\title{
RESOLUTIONS OF FACET IDEALS
}

\author{
XINXIAN ZHENG \\ FACHBEREICH MATHEMATIK UND INFORMATIK \\ UNIVERSITÄT-GHS ESSEN, 45117 ESSEN, GERMANY \\ E-MAIL: XINXIAN.ZHENG@UNI-ESSEN.DE
}

\begin{abstract}
In this paper we study the resolution of a facet ideal associated with a special class of simplicial complexes introduced by Faridi. These simplicial complexes are called trees, and are a generalization (to higher dimensions) of the concept of a tree in graph theory. We show that the Koszul homology of the facet ideal $I$ of a tree is generated by the homology classes of monomial cycles, determine the projective dimension and the regularity of $I$ if the tree is 1-dimensional, show that the graded Betti numbers of $I$ satisfy an alternating sum property if the tree is connected in codimension 1 , and classify all trees whose facet ideal has a linear resolution.
\end{abstract}

\section{INTRODUCTION}

With a simplicial complex $\Delta$ one can associate two squarefree monomial ideals: the Stanley-Reisner ideal $I_{\Delta}$ whose generators correspond to the non-faces of $\Delta$, or the facet ideal $I(\Delta)$ whose generators correspond to the facets of $\Delta$. The work of Stanley [6] has demonstrated that there are deep relations between the combinatorial properties of $\Delta$ and the algebraic properties of $I_{\Delta}$.

Facet ideals for graphs have first been considered by Villareal [7]. In this special case the facet ideal is called edge ideal, because its generators correspond to the edges of the graph. In his papers 8 and 9, Villareal has shown that the edge ideal is the appropriate algebraic object attached to a graph. Among the graphs the trees are the simplest ones. Faridi generalized in [3] and [4] the definition of tree to simplicial complexes of any dimension, and also introduced facet ideals to study trees.

In the first section of this paper we introduce the basic notions concerning trees, and give a characterization of pure trees which are connected in codimension 1 . These type of trees play an important role in the following sections.

Our goal here is to study the Koszul cycles of the facet ideal $I \subset R=K\left[x_{1}, \ldots, x_{n}\right]$ of a tree. By this we mean the cycles of the Koszul complex $K .(x, R / I)$ of $R / I$ with respect to $x_{1}, \ldots, x_{n}$. In Proposition 2.9 we show that the Koszul homology of the facet ideal of a tree has a $K$-basis with homology classes of monomial cycles as its elements. In the particular case of a 1-dimensional tree we even show that the Koszul homology of the edge ideal is generated as a $K$-algebra by the homology classes of linear cycles, see Proposition 2.12. Using this fact, in Corollary 2.13, we determine the regularity and the projective dimension of the facet ideal of a 1-dimensional tree. 
Furthermore in Theorem 2.18 we show that for the facet ideal $I$ of a 1-dimensional tree, the regularity of $R / I$ is the maximal number $j$, for which there exist $j$ edges which are pairwise disconnected.

In the third section, we consider the facet ideal $I$ of a pure tree and describe the linear part of the resolution of $R / I$, see Proposition 3.3. We call a tree whose facet ideal has a linear resolution a linear tree. In Proposition 3.9 we show that a tree is a linear tree if and only if the facet ideal of this tree is a linear quotient ideal and we classify (Theorem 3.17) all linear trees of a given dimension. Moreover in Corollary 3.10. we determine the Betti numbers of the facet ideal of a linear tree.

In the last section, we show that all trees which are connected in codimension 1 have the alternating sum property, meaning that in each linear strand of the resolution of the facet ideal except for the lowest one, the alternating sum of the graded Betti numbers is zero, and for the lowest one it is -1 .

I would like to thank Professor Jürgen Herzog for many helpful comments and discussions.

\section{FACET IDEALS}

In this section we fix the terminology, review some basic properties of graphs and introduce a notion of tree on simplicial complex given by Faridi. As a main result of this section we give a characterization of pure trees which are connected in codimension 1 . These type of trees play an important role in the following sections.

Definition 1.1. A simplicial complex $\Delta$ over a set of vertices $V=\left\{v_{1}, \ldots, v_{n}\right\}$ is a collection of subsets of $V$ with the property that $v_{i} \in \Delta$ for all $i$, and if $F \in \Delta$ then all the subsets of $F$ are also in $\Delta$ (including the empty set). An element of $\Delta$ is called a face of $\Delta$, and the dimension of a face $F$ of $\Delta$ is defined as $|F|-1$, where $|F|$ is the number of vertices of $F$. In particular, $\operatorname{dim} \emptyset=-1$. The faces of dimension 0 and 1 are called vertices and edges. The maximal faces of $\Delta$ under inclusion are called facets.

The dimension of the simplicial complex $\Delta$ is the maximal dimension of its facets, that is to say

$$
\operatorname{dim} \Delta=\max \{\operatorname{dim} F: F \in \Delta\} .
$$

We denote the simplicial complex $\Delta$ with the facets $F_{1}, \ldots, F_{q}$ by

$$
\Delta=\left\langle F_{1}, \ldots, F_{q}\right\rangle,
$$

and the facet set of $\Delta$ by $\mathcal{F}(\Delta)$. A simplicial complex $\Delta$ with only one facet is called a simplex, note that $\emptyset$ is also a simplex. A simplicial complex $\Gamma$ is called a subcomplex of $\Delta$ if $\mathcal{F}(\Gamma) \subset \mathcal{F}(\Delta)$.

Definition 1.2. Let $\Delta$ be a simplicial complex with vertices $v_{1}, \ldots, v_{n}$. Let $K$ be a field, $x_{1}, \ldots, x_{n}$ indeterminates, and $R$ the polynomial ring $K\left[x_{1}, \ldots, x_{n}\right]$. The ideal $I(\Delta) \subset R$ generated by the square-free monomials $x_{i_{1}} \cdots x_{i_{s}}$, where $\left\{v_{i_{1}}, \ldots, v_{i_{s}}\right\}$ is a facet of $\Delta$, is called the facet ideal of $\Delta$. For a 1-dimensional tree, the facet ideal is called the edge ideal. 
Definition 1.3. Let $\Delta$ be a simplicial complex of dimension $d$. Then $\Delta$ is called

(a) pure, if all of its facets have the same dimension;

(b) connected, if for any two facets $F$ and $G$ there exists a sequence of facets $F=F_{0}, \ldots, F_{n}=G$, such that $F_{i} \cap F_{i+1} \neq \emptyset$ for all $i=0, \ldots, n-1$; we call this sequence a chain between $F$ and $G$, and $n$ is called the length of this chain;

(c) connected in codimension 1 , if for any two facets $F$ and $G$ with $\operatorname{dim}(F) \geq$ $\operatorname{dim}(G)$, there exists a chain $\mathcal{C}: F=F_{0}, \ldots, F_{n}=G$ between $F$ and $G$ such that $\operatorname{dim}\left(F_{i} \cap F_{i+1}\right)=\operatorname{dim}\left(F_{i+1}\right)-1$ for all $i=0, \ldots, n-1$.

The chain $\mathcal{C}$ (in Definition $1.3(\mathrm{c})$ ) is called a proper chain. One can see that in a proper chain $\operatorname{dim} F_{i+1} \leq \operatorname{dim} F_{i}$ for $i=0, \ldots, n-1$.

Definition 1.4. A (proper) chain $\mathcal{C}$ between $F$ and $G$ is called irredundant if no subsequence of this chain except $\mathcal{C}$ itself is a (proper) chain between $F$ and $G$.

Remark 1.5. Any (proper) chain, after removing suitable facets in it, becomes an irredundant (proper) chain. In fact, let $\mathcal{C}$ be a (proper) chain between $F$ and $G$. The set of (proper) subchains of $\mathcal{C}$ is a partially ordered non-empty set. The minimal elements in this set are the irredundant (proper) chains between $F$ and $G$.

It is clear that an irredundant proper chain need not to be an irredundant chain. For example, $F_{0}=\{a, b, c\}, F_{1}=\{a, c, d\}, F_{2}=\{c, d, e\}$ is an irredundant proper chain between $F_{0}$ and $F_{2}$, but it is not an irredundant chain.

Lemma 1.6. Let $\mathcal{C}: F=F_{0}, F_{1}, \ldots, F_{n}=G$ be a proper chain between $F$ and $G$. If $\mathcal{C}$ is irredundant, then $F_{j} \neq F_{k}$ for $j \neq k$, and $F_{i} \cap F_{i+1} \nsubseteq F_{l} \cap F_{i}$ for $i=1, \ldots, n-1$, and any $l<i$.

Proof. Suppose there exists $k>j$ such that $F_{j}=F_{k}$, then $F_{0}, \ldots, F_{j}, F_{k+1}, \ldots, F_{n}$ is a proper subsequence of $\mathcal{C}$ and it is a proper chain between $F$ and $G$, a contradiction.

Thus we may now assume $F_{j} \neq F_{k}$ for $j \neq k$. Suppose there exists $i \in[n-1]$, such that $F_{i} \cap F_{i+1} \subseteq F_{l} \cap F_{i}$ for some $l<i$. Then

$$
F_{l} \cap F_{i+1} \supseteq\left(F_{l} \cap F_{i}\right) \cap\left(F_{i} \cap F_{i+1}\right)=F_{i} \cap F_{i+1},
$$

so $\operatorname{dim}\left(F_{l} \cap F_{i+1}\right) \geq \operatorname{dim}\left(F_{i} \cap F_{i+1}\right)=\operatorname{dim} F_{i+1}-1$. On the other hand, since $F_{l} \neq F_{i+1}$ both are facets, and $\operatorname{dim} F_{i+1} \leq \operatorname{dim} F_{l}$, it follows that $\operatorname{dim}\left(F_{l} \cap F_{i+1}\right) \leq \operatorname{dim} F_{i+1}-1$. Hence $\operatorname{dim}\left(F_{l} \cap F_{i+1}\right)=\operatorname{dim} F_{i+1}-1=\operatorname{dim}\left(F_{i} \cap F_{i+1}\right)$, together with $F_{l} \cap F_{i+1} \supseteq$ $F_{i} \cap F_{i+1}$, we have $F_{l} \cap F_{i+1}=F_{i} \cap F_{i+1}$. Then $F_{0}, \ldots, F_{l}, F_{i+1}, \ldots, F_{n}$ is a proper subsequence of $\mathcal{C}$, and it is a proper chain between $F$ and $G$, a contradiction.

Usually a connected graph is called a tree if it has no cycles. Now we define a very special class of trees which play an important role in Section 2.

Definition 1.7. A graph $\Gamma$ with vertex set $\left\{x, y_{1} \ldots, y_{l}\right\}, l \geq 1$, and edges $\left\{x, y_{i}\right\}$ for $i=1, \ldots, l$ is called a bouquet. We denote this bouquet by $\left(x ; y_{1} \ldots, y_{l}\right)$. The vertex $x$ is called the root, the vertices $y_{i}$ the flowers and the edges $\left\{x, y_{i}\right\}$ the stems of this bouquet.

Let $\Delta$ be a tree. If a subgraph $\Gamma$ of $\Delta$ is a bouquet, then we say $\Gamma$ is a bouquet of $\Delta$. 
In 3] Faridi introduced the notion of tree for higher dimensional simplicial complexes.

Definition 1.8. Let $\Delta$ be a simplicial complex. A facet $F$ of $\Delta$ is called a leaf if either $F$ is the only facet of $\Delta$, or there exists a facet $G$ in $\Delta, F \neq G$, such that $F \cap H \subseteq F \cap G$ for any facet $H \in \Delta, H \neq F$.

We denote the set of all facets $G \in \Delta$ with this property by $\mathcal{U}_{\Delta}(F)$ and call it the universal set of $F$ in $\Delta$.

For a facet $F$ of $\Delta$, if $x$ is a vertex of $F$ and $x$ does not belong to any other facets of $\Delta$, then we call $x$ a free vertex of $F$ in $\Delta$. It is clear that if $F$ is a leaf of $\Delta$, then $F$ has at least one free vertex. But the converse is not true, even if $\Delta$ is pure.

For example, $\Delta=\langle\{a, b, c\},\{c, d, e\},\{e, f, g\}\rangle$ is a pure simplicial complex, the facet $\{c, d, e\}$ has a free vertex $d$, but it is not a leaf.

It is easy to see that $F \in \mathcal{F}(\Delta)$ is a leaf of $\Delta$, if and only if $\langle F\rangle \cap \Gamma$ is a simplex, where $\Gamma=\langle\mathcal{F}(\Delta) \backslash\{F\}\rangle$ is the subcomplex of $\Delta$.

Lemma 1.9. Let $\mathcal{C}: F_{0}, \ldots, F_{n}$ be an irredundant chain in a simplicial complex. Then $F_{p} \cap F_{q}=\emptyset$ for any $p \in\{0, \ldots, n\}$ and any $q \neq p-1, p, p+1$. Furthermore, $F_{i}$ is not a leaf of $\Gamma=\left\langle F_{0}, \ldots, F_{n}\right\rangle$ for $i=1, \ldots, n-1$.

Proof. Suppose there exists $p \in\{0, \ldots, n\}$ and $q>p+1$ or $q<p-1$, such that $F_{p} \cap F_{q} \neq \emptyset$. We may assume that $q>p+1$, then $F_{0}, \ldots, F_{p}, F_{q}, \ldots, F_{n}$ is a chain between $F_{0}$ and $F_{n}$, a contradiction.

Suppose $F_{j}$ is a leaf of $\Gamma$ for some $j \in\{1, \ldots, n-1\}$. Since $F_{j} \cap F_{k}=\emptyset$ for any $k \neq$ $j-1, j, j+1$, we have $F_{j-1} \cap F_{j} \subseteq F_{j} \cap F_{j+1}$ or $F_{j} \cap F_{j+1} \subseteq F_{j-1} \cap F_{j}$. We may assume that $F_{j-1} \cap F_{j} \subseteq F_{j} \cap F_{j+1}$, then $F_{j} \cap F_{j+1}=\left(F_{j-1} \cap F_{j}\right) \cap\left(F_{j} \cap F_{j+1}\right) \subseteq F_{j-1} \cap F_{j+1}$. On the other hand, since $\mathcal{C}$ is a chain, $F_{j} \cap F_{j+1} \neq \emptyset$, hence $F_{j-1} \cap F_{j+1} \neq \emptyset$. It follows that $F_{0}, \ldots, F_{j-1}, F_{j+1}, \ldots, F_{n}$ is a chain. This contradicts our assumption that $\mathcal{C}$ is irredundant.

We have seen that an irredundant proper chain need not to be an irredundant chain. But as im Lemma 1.9 we also have:

Lemma 1.10. Let $\mathcal{C}: F_{0}, \ldots, F_{n}$ be an irredundant proper chain in a simplicial complex, and let $\Gamma=\left\langle F_{0}, \ldots, F_{n}\right\rangle$. Then $F_{i}$ is not a leaf of $\Gamma$, for $i=1, \ldots, n-1$.

Proof. Suppose $F_{i}$ is a leaf of $\Gamma$ for some $i \in[n-1]$. Then there exists an integer $k \neq i$ such that $F_{i} \cap F_{i+1} \subseteq F_{i} \cap F_{k}$. Since $\mathcal{C}$ is an irredundant proper chain, it follows from Lemma 1.6 that $k>i$.

For each $k \geq i+1$, we have $\operatorname{dim}\left(F_{i} \cap F_{i+1}\right)=\operatorname{dim} F_{i+1}-1 \geq \operatorname{dim} F_{k}-1 \geq$ $\operatorname{dim}\left(F_{i} \cap F_{k}\right)$. It follows that $F_{i} \cap F_{i+1}=F_{i} \cap F_{k}$. So $F_{0}, \ldots, F_{i}, F_{k}, \ldots, F_{n}$ is a proper chain between $F_{0}$ and $F_{n}$, a contradiction.

Definition 1.11 (Faridi). Let $\Delta$ be a connected simplicial complex. Then $\Delta$ is called a tree if every nonempty subcomplex of $\Delta$ has a leaf. A simplicial complex $\Delta$ with the property that every connected component is a tree is called a forest.

As a main result of this section we want to characterize when a pure tree is connected in codimension 1. For this purpose we recall the definitions of star and link of a face, see 1] [Definition 5.3.4]. 
Let $\Delta$ be a simplicial complex, and $F \in \Delta$. Then star of $F$ is the set

$$
\text { st }_{\Delta} F=\{G \in \Delta: F \cup G \in \Delta\} \text {, }
$$

and the link of $F$ is the set

$$
\mathrm{lk}_{\Delta} F=\{G \in \Delta: F \cup G \in \Delta, \quad F \cap G=\emptyset\} .
$$

To simplify notation we occasionally omit the index $\Delta$ in $\mathrm{st}_{\Delta}$ or $\mathrm{lk}_{\Delta}$. Note that $\mathrm{lk} F \subset$ st $F$, and both are simplicial complex. Furthermore, $\mathrm{lk}_{\Delta} F$ is a subcomplex of $\Delta$. Indeed one has $\mathcal{F}(\mathrm{st} G)=\{F \in \mathcal{F}(\Delta): G \subset F\}$, and $\mathcal{F}(\mathrm{lk} G)=\{F \backslash G: F \in$ $\mathcal{F}($ st $G)\}$.

We refer the reader to 1 to see that these notations are crucial in the analysis of the local cohomology of a Stanley-Reisner ring.

Proposition 1.12. Suppose that $\Delta$ is a pure tree of dimension d. Then the following are equivalent:

(i) for all $G \in \Delta$ with $\operatorname{dim} G \leq d-2, \mathrm{lk} G$ is connected;

(ii) $\Delta$ is connected in codimension 1 .

Proof. (i) $\Rightarrow($ ii): Suppose $\Delta$ is not connected in codimension 1 . Then there exists $F, H \in \mathcal{F}(\Delta)$ such that there is no proper chain between $F$ and $H$. Since $\Delta$ is a tree, it is connected, and hence there exists a chain $F=H_{0}, H_{1}, \ldots, H_{q}=H$ between $F$ and $G$. Let $a=\min \left\{\operatorname{dim}\left(H_{i} \cap H_{i+1}\right): i=0, \ldots, q-1\right\}$. Since this chain is not proper we have $0 \leq a<d-1$. We may assume that there is no other chain $F=K_{0}, \ldots, K_{p}=H$ in $\Delta$, such that $\min \left\{\operatorname{dim}\left(K_{i} \cap K_{i+1}\right): i=0, \ldots, p-1\right\}>a$, otherwise we take this chain instead of $H_{0}, \ldots, H_{q}$. Let $\left\{i_{1}, \ldots, i_{m}\right\} \subseteq\{0, \ldots, q\}$ be the subset such that $\operatorname{dim}\left(H_{i_{j}} \cap H_{i_{j}+1}\right)=a$, we know that $\left\{i_{1}, \ldots, i_{m}\right\} \neq \emptyset$. By the choice of our chain there must exist $j \in\{1, \ldots, m\}$ such that there is no chain $H_{i_{j}}=$ $E_{0}, E_{1}, \ldots, E_{s}=H_{i_{j}+1}$ in $\Delta$ such that $\min \left\{\operatorname{dim}\left(E_{i} \cap E_{i+1}\right): i=0, \ldots, s-1\right\}>a$.

Let $G=H_{i_{j}} \cap H_{i_{j}+1}$, then $\operatorname{dim} G=a<d-1$. We claim that lk $G$ is not connected. In fact, if $1 \mathrm{k} G$ is connected, then there exists a chain $H_{i_{j}}=D_{0}, D_{1}, \ldots, D_{l}=H_{i_{j}+1}$ in st $G$ such that $\left(D_{i} \backslash G\right) \cap\left(D_{i+1} \backslash G\right) \neq \emptyset$, for any $i=1, \ldots, l-1$. This implies that $\operatorname{dim}\left(D_{i} \cap D_{i+1}\right)>a$ for any $i=1, \ldots, l-1$, a contradiction to the choice of $j$.

(ii) $\Rightarrow$ (i): Suppose there exists $G \in \Delta$ with $\operatorname{dim} G \leq d-2$, such that $\mathrm{lk} G$ is not connected. Then there exist facets $F$ and $H$ in st $G$ such that there is no chain between $F \backslash G$ and $H \backslash G$ in lk $G$.

Since $\Delta$ is connected in codimension 1, there exists an irredundant proper chain $F=H_{0}, H_{1}, \ldots, H_{r}=H$ between $F$ and $H$. Since $\operatorname{dim} G \leq d-2$, it follows that $\left(H_{i} \cap H_{i+1}\right) \backslash G \neq \emptyset, i=0, \ldots, n-1$. Therefore not all $H_{i}$ belong to st $G$, because otherwise $F \backslash G=H_{0} \backslash G, H_{1} \backslash G, \ldots, H_{r} \backslash G=H \backslash G$ would be a chain between $F \backslash G$ and $H \backslash G$ in $\operatorname{lk} G$.

Let $l=\min \left\{j \in\{0, \ldots, r\}: H_{j+1} \notin \mathrm{st} G\right\}$, and let $m=\min \left\{j \in\{l+2, \ldots, n\}: H_{j}\right.$ $\in$ st $G\}$. Now consider the sequence of facets $H_{l}, \ldots, H_{m}$, it is an irredundant proper chain between $H_{l}$ and $H_{m}$, and $H_{l}, H_{m} \in$ st $G, H_{l+1}, \ldots, H_{m-1} \notin$ st $G$.

Take the subcomplex $\Gamma=\left\langle H_{l}, \ldots, H_{m}\right\rangle$ of $\Delta$. Then this subcomplex has no leaf, and so $\Delta$ is not a tree, a contradiction. Indeed, since $H_{l}, \ldots, H_{m}$ it is an irredundant proper chain, it follows from Lemma 1.10 that $H_{i}$ is not a leaf for $i=l+1, \ldots, m-1$. 
Now consider the facet $H_{l}$, and let $H_{l} \cap H_{l+1}=H$. Then $H$ is a face of $\Delta$ with dimension $d-1$. Let $\{w\}=H_{l} \backslash H_{l+1}$ and $\{u\}=H_{l+1} \backslash H_{l}$. Since $H_{l+1} \notin$ st $G$, $G \not \subset H$. On the other hand, $H_{l} \in$ st $G$, we must have $w \in G$. From $H_{m} \in$ st $G$ we know $w \in H_{m}$. That is to say $H_{l}$ has no free vertex in $\Gamma$, hence $H_{1}$ is not a leaf of $\Gamma$. With the same argument we can show that $H_{m}$ is not a leaf of $\Gamma$.

Corollary 1.13. Let $\Delta$ be a pure tree of dimension d and connected in codimension 1. Then for any facet $F$ of $\Delta$, all the facets of $\langle F\rangle \cap\langle\mathcal{F}(\Delta) \backslash\{F\}\rangle$ are of dimension $d-1$.

Proof. Suppose there exists a facet $F$ of $\Delta$, such that $\langle F\rangle \cap\langle\mathcal{F}(\Delta) \backslash\{F\}\rangle$ is not pure of dimension $d-1$. Then there exists $H \in \mathcal{F}(\Delta)$ such that $F \cap H=G$ with $\operatorname{dim} G \leq d-2$ and $G \not \subset F \cap H^{\prime}$ for all $H^{\prime} \in \mathcal{F}(\Delta) \backslash\{F\}$.

We claim $\mathrm{lk} G$ is not connected. In fact, assume $\mathrm{lk} G$ is connected, then, since $F \in \mathcal{F}(\operatorname{st} G)$ if and only if $F \backslash G \in \mathcal{F}(\operatorname{lk} G)$, there exists a sequence of facets $F=F_{0}, F_{1}, \ldots, F_{r}=H$ in st $G$ such that $\left(F_{i} \backslash G\right) \cap\left(F_{i+1} \backslash G\right) \neq \emptyset$ for $i=0, \ldots, r-1$. We may assume $F_{1} \neq F$. Since $(F \backslash G) \cap\left(F_{1} \backslash G\right) \neq \emptyset, G$ is a proper subset of $F \cap F_{1}$, a contradiction.

Now Proposition 1.12 implies $\Delta$ is not connected in codimension 1, a contradiction to our hypothesis.

Remark 1.14. Let $\Delta$ be a pure tree of dimension $d$. Even if for any facet $F$ of $\Delta$, all the facets of $\langle F\rangle \cap\langle\mathcal{F}(\Delta) \backslash\{F\}\rangle$ are of dimension $d-1, \Delta$ may not be connected in codimension 1.

For example, $\Delta=\langle\{a, b, c\},\{b, c, d\},\{c, e, f\},\{c, f, g\}\rangle$ is pure of dimension 2 , and for any facet $F$ of $\Delta$, the facets of $\langle F\rangle \cap\langle\mathcal{F}(\Delta) \backslash\{F\}\rangle$ are of dimension 1, but $\Delta$ is not connected in codimension 1 .

However we have:

Corollary 1.15. Let $\Delta$ be a pure tree of dimension $d$ and connected in codimension $1, F$ a facet of $\Delta$. Then $\Gamma=\langle\mathcal{F}(\Delta) \backslash\{F\}\rangle$ is connected in codimension 1 if and only if $F$ is a leaf of $\Delta$.

Proof. Assume $F$ is a leaf of $\Delta$. By Lemma 1.10, for any irredundant proper chain $\mathcal{C}: F_{0}, \ldots, F_{l}$ in $\Delta, F \neq F_{i}$ for any $i \in[l-1]$. Hence $\Gamma$ is connected in codimension 1 .

Now assume $\Gamma$ is connected in codimension 1. By Corollary 1.13, $\langle F\rangle \cap \Gamma$ is a pure simplicial complex of dimension $d-1$. Assume $F$ is not a leaf of $\Delta$. Then there exist two facets $H_{1}$ and $H_{2}$ in $\Gamma$ such that $\operatorname{dim}\left(F \cap H_{i}\right)=d-1, i=1,2$. Let $G=H_{1} \cap H_{2}$. Then $\operatorname{dim} G=d-2$. We may assume $H_{1}=G \cup\left\{x_{1}, x_{2}\right\}$ and $H_{2}=G \cup\left\{x_{3}, x_{4}\right\}$ and $F=G \cup\left\{x_{1}, x_{4}\right\}$, where $x_{i}$ are vertices. Since $\Gamma$ is a pure tree and connected in codimension 1 , by Proposition $1.12, \mathrm{k}_{\Gamma} G$ is connected. Let $\left\{x_{1}, x_{2}\right\}=H_{1} \backslash G=F_{1} \backslash G, \ldots, F_{l} \backslash G=H_{2} \backslash G=\left\{x_{3}, x_{4}\right\}$ be an irredundant chain between $\left\{x_{1}, x_{2}\right\}$ and $\left\{x_{3}, x_{4}\right\}$ in $\mathrm{lk}_{\Gamma} G$. Then the subcomplex $\left\langle F, F_{1}, \ldots, F_{l}\right\rangle$ of $\Delta$ has no leaf, a contradiction. Indeed, each vertex in $\left\langle F, F_{1}, \ldots, F_{l}\right\rangle$ belongs to at least two facets of this subcomplex. 
Another consequence of Corollary 1.13 is

Proposition 1.16. Let $\Delta$ be a pure tree which is connected in codimension 1, and has more than one facet. Then $\Delta$ has at least two leaves.

Proof. Let $\operatorname{dim} \Delta=d$. Suppose $\Delta$ has only one leaf. Let $F_{1}$ be this leaf. Since $\Delta$ is connected and has more than one facet, there exists a facet $G$ such that $F_{1} \cap G \neq \emptyset$. Since $\Delta$ is pure it follows from Corollary 1.13 that there exists a facet $F_{2}$, such that $F_{1} \cap G \subseteq F_{1} \cap F_{2}$ and $\operatorname{dim}\left(F_{1} \cap F_{2}\right)=d-1$. Let $F_{2} \backslash F_{1}=\{x\}$. Since $F_{2}$ is not a leaf, there exists a facet $H$, such that $\{x\} \subseteq F_{2} \cap H$. Again by Corollary 1.13 there exists a facet $F_{3}$, such that $F_{2} \cap H \subseteq F_{2} \cap F_{3}$ and $\operatorname{dim}\left(F_{2} \cap F_{3}\right)=d-1$. It is clear that $F_{3} \neq F_{1}$. Since $F_{3}$ is not a leaf, by the same reason there exists a facet $F_{4} \neq F_{2}$, and $\operatorname{dim}\left(F_{3} \cap F_{4}\right)=d-1$, and so on. Since there are only finitely many facets, there exist integers $i$ and $j$ with $j<i-1$ such that $F_{i}=F_{j}$. If $F_{i-1} \cap F_{i} \neq F_{j} \cap F_{j+1}$, then the subcomplex $\left\langle F_{j}, \ldots, F_{i-1}\right\rangle$ has no leaf. If $F_{i-1} \cap F_{i}=F_{j} \cap F_{j+1}$, then the subcomplex $\left\langle F_{j+1}, \ldots, F_{i-1}\right\rangle$ has no leaf. This contradicts our assumption that $\Delta$ is a tree.

By definition, in a simplicial complex $\Delta$ which is connected in codimension 1 , for any two facets $F$ and $G$, there exists an irredundant proper chain between $F$ and $G$. For a pure tree we even have

Proposition 1.17. Let $\Delta$ be a pure tree and connected in codimension 1 . Then for any two facets $F$ and $G$, there exists a unique irredundant proper chain between $F$ to $G$.

Proof. Suppose $\mathcal{C}: F=F_{0}, \ldots, F_{n}=G$ and $\mathcal{C}^{\prime}: F=G_{0}, \ldots, G_{m}=G$ are two different irredundant proper chains between $F$ and $G$. Let $l=\min \left\{j\right.$ : such that $F_{j} \neq$ $\left.G_{j}\right\}$, and $k=\min \left\{i: i \geq j+1\right.$ and $F_{i}=G_{t}$ for some $\left.t\right\}$. Then, since $\mathcal{C}$ and $\mathcal{C}^{\prime}$ both are irredundant, $t>l$ and $G_{t} \neq G_{i}$ for any $i \neq t$. Let $\Gamma$ be a subcomplex of $\Delta$, such that $F_{l}, \ldots, F_{k-1}, G_{l}, \ldots, G_{t-1} \in \Gamma$; if $F_{l-1} \cap F_{l} \neq G_{l-1} \cap G_{l}$, then let $F_{l-1} \in \Gamma$; if $F_{k-1} \cap F_{k} \neq G_{t-1} \cap G_{t}$, then let $F_{k} \in \Gamma$; and there are no other facet in $\Gamma$. By Lemma 1.10 one can easily check that $\Gamma$ has no leaf, a contradiction since $\Delta$ is a tree.

According to this proposition, we give the following definition:

Definition 1.18. Let $\Delta$ be a pure tree and connected in codimension 1 . For any two facets $F$ and $G$, the length of the unique irredundant proper chain between $F$ and $G$ is called the distance between $F$ and $G$, and denoted by $\operatorname{dist}(F, G)$.

We call $\max \{\operatorname{dist}(F, G): F$ and $G$ are two facets of $\Delta\}$ the diameter of $\Delta$.

If $\Delta$ is a pure forest and each connected component is connected in codimensiom 1 , then for any two facets $F$ and $G$ which lie in two different components, we set $\operatorname{dist}(F, G)=\infty$.

Remark 1.19. Let $\Delta$ be a pure tree and connected in codimension 1 with diameter $l$, and $F_{0}, \ldots, F_{l}$ an irredundant proper chain of length $l$ in $\Delta$. Then $F_{0}$ and $F_{l}$ are leaves of $\Delta$. 
Indeed, since $F_{l} \ldots, F_{0}$ is also an irredundant proper chain of length $l$, we only need to show $F_{0}$ is a leaf of $\Delta$. Let $d=\operatorname{dim} \Delta$. By Corollary 1.13. $\left\langle F_{0}\right\rangle \cap\left\langle\mathcal{F}(\Delta) \backslash\left\{F_{0}\right\}\right\rangle$ is a pure simplicial complex of dimension $d-1$. Suppose $F_{0}$ is not a leaf. Then there exists a facet $F$ of $\Delta$ such that $\operatorname{dim}\left(F_{0} \cap F\right)=d-1$ and $F_{0} \cap F \neq F_{0} \cap F_{1}$. Hence $F, F_{0} \ldots, F_{l}$ is an irredundant chain in $\Delta$ with length $l+1$, a contradiction.

Sometimes we consider a kind of simplicial complex which need not to be a tree, but has some nice properties like a tree, we call it a quasi-tree.

A connected simplicial complex $\Delta$ is called a quasi-tree, if there exists an order $F_{1}, \ldots, F_{n}$ of the facets, such that $F_{i}$ is a leaf of $\left\langle F_{1}, \ldots, F_{i}\right\rangle$ for each $i=1, \ldots, n$. Such an order is called a leaf order. A simplicial complex $\Delta$ with the property that every connected component is a quasi-tree is called a quasi-forest.

A tree is a quasi-tree, hence for any tree there exists a leaf order of facets. But a quasi-tree need not to be a tree.

For example, $\Delta=\langle\{a, b, c\},\{b, c, d\},\{c, d, e\},\{b, d, f\}\rangle$ is a quasi-tree, but it is not a tree, because the subcomplex $\langle\{a, b, c\},\{c, d, e\},\{b, d, f\}\rangle$ has no leaf.

\section{On the Koszul Cycles of the facet ideal of a tree}

In the remaining sections $R=K\left[x_{1}, \ldots, x_{n}\right]$ denotes the polynomial ring in $n$ indeterminates over the field $K$. Let $M$ be an $R$-module, we denote the Koszul complex $K_{.}(x, M)$ of $M$ with respect to the sequence $x_{1}, \ldots, x_{n}$ by $K .(M)$, and for the modules of Koszul cycles, Koszul boundaries and the Koszul homology we write $Z$. $(M), B$. $(M)$ and $H_{\text {. }}(M)$, respectively.

For simplicity, in the remaining sections all simplicial complexes will have the variables $x_{1}, \ldots, x_{n}$ as vertices. For a facet $F=\left\{x_{i_{1}}, \ldots, x_{i_{s}}\right\}$ in $\Delta$, we denote by $f=x_{i_{1}} \cdots x_{i_{s}}$ (in small letter) the monomial in $R$ corresponding to $F$.

For the proof of the main result of this section which describes the Koszul cycles of certain monomial ideals, we need the following general result on the shifts in the resolution of a $\mathbb{Z}^{n}$-graded module: Let $M$ be a finite $\mathbb{Z}^{n}$-graded $R$-module with minimal $\mathbb{Z}^{n}$-graded free resolution

$$
\cdots \longrightarrow \bigoplus_{a \in \mathbb{Z}^{n}} R(-a)^{b_{1 a}} \longrightarrow \bigoplus_{a \in \mathbb{Z}^{n}} R(-a)^{b_{0 a}} \longrightarrow M \longrightarrow 0 .
$$

The numbers $b_{i a}$ are called the multigraded Betti numbers of $M$.

We define the support of an element $a \in \mathbb{Z}^{n}$ to be the set $\operatorname{supp} a=\left\{i: a_{i} \neq 0\right\}$. Without ambiguity, we may set $\operatorname{supp} x^{a}=\operatorname{supp} a$ for any non-zero monomial. We set $\mathbb{Z}_{+}^{n}=\left\{a \in \mathbb{Z}^{n}: a_{i} \geq 0\right.$ for all $\left.i=1, \ldots, n\right\}$. Then we have

Lemma 2.1. Let $M$ be a torsion-free $\mathbb{Z}^{n}$-graded $R$-module, and $y_{1}, \ldots, y_{s}$ a minimal homogeneous generating system of $M$. Suppose that $\operatorname{supp}\left(\operatorname{deg}\left(y_{i}\right)\right) \subseteq \mathbb{Z}_{+}^{n}$ and $t \notin$ $\operatorname{supp}\left(\operatorname{deg}\left(y_{i}\right)\right)$ for $i=1, \ldots, s$. Then $t \notin \operatorname{supp}(a)$ for all non-zero multigraded Betti numbers $b_{i a}$ of $M$.

Proof. We prove the assertion by induction on $\operatorname{proj} \operatorname{dim}(M)$. If $\operatorname{proj} \operatorname{dim}(M)=0$, then the assertion is obvious. Now assume $\operatorname{proj} \operatorname{dim}(M)>0$, and let $F$. be the minimal multigraded free $R$ resolution of $M$, and $\varepsilon: F_{0} \rightarrow M$ the augmentation map. 
Obviously $t \notin \operatorname{supp}(a)$ for all $b_{0 a}$ which are non-zero. Let $e_{1}, \ldots, e_{s}$ be a multigraded basis of $F_{0}$ with $\varepsilon\left(e_{i}\right)=y_{i}$ for $i=1, \ldots, s$, and let $z=\sum c_{i} e_{i}$ be a homogeneous element in a minimal homogeneous set of generators of $\operatorname{Ker}(\varepsilon)$. Then $\operatorname{deg}(z)=\operatorname{deg}\left(c_{i}\right)+\operatorname{deg}\left(e_{i}\right)$ for $i=1, \ldots, s$. By assumption we have $\left[\operatorname{deg}\left(e_{i}\right)\right]_{t}=0$ for $i=1, \ldots, s$. Suppose $t \in \operatorname{supp}(\operatorname{deg}(z))$, then $\left[\operatorname{deg}\left(c_{i}\right)\right]_{t}>0$ for all $i$ with $c_{i} \neq 0$. This implies that there exist $c_{i}^{\prime} \in R$ such that $c_{i}=x_{t} c_{i}^{\prime}$ for all $i$. So we have $z=x_{t} \sum c_{i}^{\prime} e_{i}$ and so $x_{t} \sum c_{i}^{\prime} y_{i}=0$. Since $M$ is a torsion-free module, it follows that $\sum c_{i}^{\prime} y_{i}=0$, and hence $\sum c_{i}^{\prime} e_{i} \in \operatorname{Ker}(\varepsilon)$. That is to say, $z \in \mathfrak{m} \operatorname{Ker}(\varepsilon)$, where $\mathfrak{m}=\left(x_{1}, \ldots, x_{n}\right)$, contradicting the assumption that $z$ belongs to a minimal homogeneous generating system of $\operatorname{Ker}(\varepsilon)$. Therefore $t$ does not belong to the support of any element in a minimal set of generators of $\operatorname{Ker}(\varepsilon)$. Since $\operatorname{Ker}(\varepsilon)$ is torsion free and $\operatorname{proj} \operatorname{dim}(\operatorname{Ker}(\varepsilon))<\operatorname{proj} \operatorname{dim}(M)$, the lemma follows from our induction hypothesis.

Let $J$ be a monomial ideal. As usual we denote by $G(J)$ the unique minimal set of monomial generators of $J$. We put $[n]$ to be the set $\{1, \ldots, n\}$.

Lemma 2.2. Let $I$ and $J$ be monomial ideals in $R$ with $G(I)=\left\{f_{1}, \ldots, f_{m}\right\}$ and $G(J)=\left\{f_{1}, \ldots, f_{m-1}\right\}$, and let $b$ be the multidegree of $f_{m}$. If there exists $t \in[n]$, such that $t \in \operatorname{supp}(b)$, but $t \notin \operatorname{supp}\left(\operatorname{deg}\left(f_{i}\right)\right)$ for $i=1, \ldots, m-1$. Then $t \in \operatorname{supp}(a)$ for all a with $b_{i a}(R /(J: I)(-b)) \neq 0$.

Proof. Let $F$. be the minimal $\mathbb{Z}^{n}$-graded free resolution of $R /(J: I)$, then $F$. $(-b)$ is the minimal $\mathbb{Z}^{n}$-graded free resolution of $R /(J: I)(-b)$. Since $t \notin \operatorname{supp}\left(\operatorname{deg}\left(f_{i}\right)\right)$ for $1 \leq i \leq m-1, t$ is not in the support of the elements of $G(J: I)=G\left(J: f_{m}\right)$. This is because $G\left(J: f_{m}\right)$ is a subset of $\left\{f_{1} / \operatorname{gcd}\left(f_{1}, f_{m}\right), \ldots, f_{m-1} / \operatorname{gcd}\left(f_{m-1}, f_{m}\right)\right\}$. Applying Lemma 2.1 to $J: I$, we have that $t \notin \operatorname{supp}(a)$ for all $b_{i a}(J: I) \neq 0$. Hence $t \notin \operatorname{supp}(a)$ for all $b_{i a}(R /(J: I)) \neq 0$. Since $b_{i a}(R /(J: I)(-b))=b_{i, a-b}(R /(J: I))$, we have $t \notin \operatorname{supp}(a-b)$, for any $b_{i a}(R /(J: I)(-b)) \neq 0$. But $t \in \operatorname{supp}(b)$, hence $t \in \operatorname{supp}(a)$.

Theorem 2.3. Let $J \subset R$ be a monomial ideal, $f \in R \backslash J$ a monomial and let $I=(J, f)$. Suppose that there exists an integer $t$ such that $x_{t}$ divides $f$, but $x_{t}$ does not divide any $g \in G(J)$. Then for all $i>0$ there exist short exact sequences

$$
0 \longrightarrow H_{i}(R / J) \longrightarrow H_{i}(R / I) \stackrel{\delta}{\longrightarrow} H_{i-1}(R /(J: I)(-b)) \longrightarrow 0,
$$

where $b$ is the multidegree of $f$, and for each homology class $[z] \in H_{i-1}(R /(J$ : $I)(-b))$ the homology class $\left[(-1)^{\operatorname{deg} z} z \wedge\left(f / x_{t}\right) e_{t}\right]$ is a preimage of $[z]$ under $\delta$.

Proof. From the exact sequence

$$
0 \longrightarrow R /(J: I)(-b) \longrightarrow R / J \longrightarrow R / I \longrightarrow 0,
$$

we get the long exact sequence

$$
\begin{gathered}
\cdots \longrightarrow H_{i}(R /(J: I)(-b)) \longrightarrow H_{i}(R / J) \longrightarrow H_{i}(R / I) \\
\stackrel{\delta}{\longrightarrow} H_{i-1}(R /(J: I)(-b)) \longrightarrow,
\end{gathered}
$$


Let $F$. be the minimal $\mathbb{Z}^{n}$-graded free resolution of $R / J$, then

$$
\operatorname{Tor}_{i}^{R}(K, R / J)=\bigoplus K(-a)^{b_{i a}(R / J)}=H_{i}(R / J),
$$

and

$$
\operatorname{Tor}_{i}^{R}(K, R /(J: I)(-b))=\bigoplus K(-a)^{b_{i a}(R /(J: I)(-b))}=H_{i}(R /(J: I)(-b)) .
$$

From Lemma 2.1 and Lemma 2.2 we know that $t \notin \operatorname{supp}(a)$ for all $b_{i a}(R / J) \neq 0$, but $t \in \operatorname{supp}(a)$ for all $b_{i a}(R /(J: I)(-b)) \neq 0$. Since $H_{i}(R /(J: I)(-b)) \rightarrow H_{i}(R / J)$ is a homogeneous homomorphism, it must be the zero map. Hence we have the exact sequence as required.

To show $\left[(-1)^{\operatorname{deg} z} z \wedge\left(f / x_{t}\right) e_{t}\right]$ is the preimage of $[z]$, we only need to show

$$
d\left((-1)^{\operatorname{deg} z} z \wedge\left(f / x_{t}\right) e_{t}\right)=f z \quad \text { in } \quad K .(R / J) .
$$

In fact, $d\left((-1)^{\operatorname{deg} z} z \wedge\left(f / x_{t}\right) e_{t}\right)=(-1)^{\operatorname{deg}(z)} d(z) \wedge\left(\left(f / x_{t}\right) e_{t}\right)+f z$. Now since $z \in$ $Z_{i-1}(R /(J: I)(-b))$, it follows that $d(z) \in(J: I) K_{i-2}(R)$, and hence $x_{t}\left(f / x_{t}\right) d(z)=$ $f d(z) \in J K_{i-2}(R)$. Since $x_{t}$ does not divide any $g \in G(J)$, we have $J=J: x_{t}$, and so $\left(f / x_{t}\right) d(z) \in J K_{i-2}(R)$. Hence $d(z) \wedge\left(f / x_{t}\right) e_{t} \in J K_{i-1}(R)$. That is to say, $d(z) \wedge\left(f / x_{t}\right) e_{t}=0$ in $K_{i-1}(R / J)$.

Corollary 2.4. Let $L \subset R$ be a graded ideal, and $x_{i_{1}}, \ldots, x_{i_{s}}$ a regular sequence on $R / L$. If $B$ is a $K$-basis of $H .(R / L)$, then $\left\{\left[z \wedge e_{I}\right]:[z] \in B, \quad I \subset\left\{i_{1}, \ldots, i_{s}\right\}\right\}$ is a $K$-basis of $H_{\cdot}\left(R / L+\left(x_{i_{1}}, \ldots, x_{i_{s}}\right)\right)$.

Proof. We may assume that $s=1$. The general case is done by induction on $s$. Since $x_{i_{1}}$ is regular on $R / L, x_{i_{1}}$ does not divide any $g \in G(L)$. Therefore the result follows from Theorem 2.3

Corollary 2.5. Let $I, I^{\prime}$ be monomial ideals in $R$ with $G(I)=\left\{f_{1}, \ldots, f_{m}, \ldots, f_{l}\right\}$ and $G\left(I^{\prime}\right)=\left\{f_{1}, \ldots, f_{m}\right\}$. If for any $i \geq m+1$ there exists a variable which divides $f_{i}$ but does not divide $f_{j}$ for any $j<i$. Then the map $H_{i}\left(R / I^{\prime}\right) \rightarrow H_{i}(R / I)$ is injective.

Proof. The statement follows immediately from Theorem 2.3 by induction on $l-m$.

Corollary 2.6. Let $R, I, J$ be as in Theorem 2.3, Then we have

$$
b_{i a}(R / I)=b_{i a}(R / J)+b_{i-1, a-b}(R /(J: I)) \text { for all } i>0 \text { and } a \in \mathbb{Z}^{n} .
$$

For another main result of this section, we need the following concept:

Definition 2.7. Let $I$ be a monomial ideal of $R$. A cycle $z$ of $K$. $(R / I)$ is called a monomial cycle if there exists $L \subset[n]$ and a monomial $f$, such that $z=f e_{L}$.

Even if $I$ is a square-free monomial ideal, $H_{.}(R / I)$ may not be generated by homology classes of monomial cycles. For example, let $R=K\left[x_{1}, x_{2}, x_{3}, x_{4}\right]$ and $I=\left(x_{1} x_{2}, x_{2} x_{3}, x_{3} x_{4}, x_{4} x_{1}\right)$. Then $z=x_{1} e_{2} \wedge e_{3} \wedge e_{4}+x_{3} e_{1} \wedge e_{2} \wedge e_{4}$ is a cycle, but $z$ is not homologous to a monomial cycle. In fact, a boundary $b \in B_{3}(R / I)$ is of the form $d\left(f e_{1} \wedge e_{2} \wedge e_{3} \wedge e_{4}\right)$. So $z$ can not be a monomial cycle.

However for the facet ideal $I$ of a forest, we have $H_{\text {. }}(R / I)$ is generated by homology classes of monomial cycles. To prove this we need the following lemma. 
Lemma 2.8. Let $\Delta$ be a forest and $I$ its facet ideal. If $F$ is any facet of $\Delta$ and $J$ is the ideal generated by $G(I) \backslash\{f\}$. Then the simplicial complex $\Delta^{\prime}$ with facet ideal $J: I$ is again a forest.

Proof. Note that $\mathcal{F}\left(\Delta^{\prime}\right)$ is a subset of $\{G \backslash F: G \in \mathcal{F}(\Delta)\}$. Suppose $\Delta^{\prime}$ is not a forest. Then there exist facets $F_{1}, \ldots, F_{p}$ of $\Delta$, such that the subcomplex $\left\langle F_{1} \backslash F, \ldots, F_{p} \backslash F\right\rangle$ of $\Delta^{\prime}$ has no leaf. Since $\Delta$ is a forest, the subcomplex $\left\langle F_{1}, \ldots, F_{p}\right\rangle$ has a leaf $F_{i}$. Hence there exists a integer $k \in\{1, \ldots, p\}$ and $k \neq i$, such that $F_{j} \cap F_{i} \subseteq F_{k} \cap F_{i}$ for any $j \neq i$. Therefore $\left(F_{j} \cap F_{i}\right) \backslash F \subseteq\left(F_{k} \cap F_{i}\right) \backslash F$ for any $j \neq i$, and hence $\left(F_{j} \backslash F\right) \cap\left(F_{i} \backslash F\right) \subseteq\left(F_{k} \backslash F\right) \cap\left(F_{i} \backslash F\right)$ for any $j \neq i$. So $F_{i} \backslash F$ is a leaf of $\left\langle F_{1} \backslash F, \ldots, F_{p} \backslash F\right\rangle$, a contradiction.

Proposition 2.9. Let $\Delta$ be a forest and $I$ its facet ideal. Then $H_{r}(R / I)$ has the K-basis

$$
\mathcal{M}_{r}=\left\{\left[f e_{i_{1}} \wedge \cdots \wedge e_{i_{r}}\right]: f e_{i_{1}} \wedge \cdots \wedge e_{i_{r}} \text { is a monomial cycle. }\right\}
$$

Proof. Let $\Delta=\left\langle F_{1} \ldots, F_{m}\right\rangle$ where $F_{1} \ldots, F_{m}$ is a leaf order. We prove the assertion by induction on $m$. The case $m=1$ is trivial. Since $F_{m}$ is a leaf we may assume that $f_{m}=h x_{t}$, where $h$ is a monomial and $x_{t} \in F_{m} \backslash \bigcup_{j<m} F_{j}$. By Theorem 2.3, we have short exact sequences

$$
0 \longrightarrow H_{r}(R / J) \longrightarrow H_{r}(R / I) \longrightarrow H_{r-1}(R /(J: I)(-b)) \longrightarrow 0,
$$

where $J=\left(f_{1}, \ldots, f_{m-1}\right)$ and $b$ is the multidegree of $f_{m}$. By Lemma 2.8, $J: I$ is a facet ideal of a forest and it has at most $m-1$ facets. Again use Theorem 2.3 we have $[z],\left[z^{\prime} \wedge\left(f_{m} / x_{t}\right) e_{t}\right]$ are basis elements of $H_{r}(R / I)$, where $[z]$ and $\left[z^{\prime}\right]$ are basis elements of $H_{r}(R / J)$ and $H_{r-1}(R /(J: I)(-b))$, respectively. And by induction hypothesis $z$ and $z^{\prime}$ can be choosen as monomial cycles.

Definition 2.10. Let $I$ be a monomial ideal and let $d$ be the least degree of its generators. A monomial cycle $z=f e_{L}$ in $K \cdot(R / I)$ is called linear if $f$ is a monomial of degree $d-1$.

Remark 2.11. Let $\Delta$ be a 1-dimensional forest with edge ideal $I$. Then the linear monomial cycles are of the form

$$
x_{l} e_{l_{1}} \wedge \cdots \wedge e_{l_{r}},
$$

where $\left\{x_{l}, x_{l_{i}}\right\}$ is an edge of $\Delta, i=1, \ldots, r$. Hence it follows from Proposition 2.9] that the set

$$
\mathcal{B}_{r}=\left\{[z(b)]: b=\left(x_{l} ; x_{l_{1}}, \ldots, x_{l_{r}}\right) \text { is a bouquet of } \Delta \text { with } r \text { flowers }\right\}
$$

is a $K$-basis of $H_{r}(R / I)_{r+1}$, where $z(b)=x_{l} e_{l_{1}} \wedge \cdots \wedge e_{l_{r}}$.

Proposition 2.12. Let $\Delta$ be a forest of dimension 1 and $I$ its facet ideal. Then as a K-algebra, $H_{.}(R / I)$ is generated by the homology classes of linear monomial cycles.

Proof. Let $f e_{L}$ be an arbitrary monomial cycle, and let $i \in L$. Then $f x_{i} \in I$, and hence there exists a generator $f_{1} \in G(I)$ such that $f x_{i}=f_{1} g$. Since $f \notin I$, we conclude that $x_{i}$ divides $f_{1}$. Then $f=\left(f_{1} / x_{i}\right) g$. Now let $L_{1}=\left\{l \in L:\left(f_{1} / x_{i}\right) x_{l} \in\right.$ 
$I\}$, and $L_{2}=L \backslash L_{1}$. Note that $i \in L_{1}$ and that $f e_{L}=\left(f_{1} / x_{i}\right) e_{L_{1}} \wedge g e_{L_{2}}$, where $\left(f_{1} / x_{i}\right) e_{L_{1}}$ is a linear cycle. If $g=1$, then $f e_{L}$ is a linear cycle, and if $g \neq 1$ but $L_{2}=\emptyset$, then $f e_{L}$ is a boundary. Thus we may assume that $g \neq 1$ and $L_{2} \neq \emptyset$, and have to show that $g e_{L_{2}}$ is a cycle. Then we can proceed by induction on the degree of $f$.

Suppose $g x_{s} \notin I$ for some $s \in L_{2}$. Since $f=\left(f_{1} / x_{i}\right) g$ we have $\left(\left(f_{1} / x_{i}\right) g\right) x_{s} \in I$. Let $f_{1} / x_{i}=x_{r}$. By the choice of $L_{2}$ it follows that $x_{r} x_{s} \notin I$. Therefore there must exist $x_{t}$ dividing $g$ such that $x_{t} x_{s} \in I$. This implies $g x_{s} \in I$, a contradiction.

Corollary 2.13. Let $\Delta$ be a 1-dimensional forest with edge ideal I. Then

(i) $\operatorname{reg}(R / I)$ is the maximal number $j$ for which there exist linear monomial cycles $z_{i}$ such that $\left[z_{1}\right] \cdots\left[z_{j}\right] \neq 0$;

(ii) $\operatorname{pd}(R / I)$ is the maximum among the sums $\sum_{i=1}^{j} k_{i}$ for which there exist linear cycles $z_{i} \in Z_{k_{i}}(R / I)$ such that $\left[z_{1}\right] \cdots\left[z_{j}\right] \neq 0$.

Proposition 2.14. Let $\Delta$ be a 1-dimensional forest with edge ideal I, and let $b_{1}=\left(x_{q_{1}} ; x_{q_{11}}, \ldots, x_{q_{1_{1}}}\right), \ldots, b_{l}=\left(x_{q_{l}} ; x_{q_{l 1}}, \ldots, x_{q_{l_{l}}}\right)$ be bouquets in $\Delta$. Then the following statements are equivalent:

(i) $\left[z\left(b_{1}\right)\right] \cdots\left[z\left(b_{l}\right)\right] \neq 0$.

(ii) The set of bouquets $b_{1}, \ldots, b_{l}$ satisfies the following conditions:

(a) All vertices occurring in these bouquets are pairwise distinct.

(b) The roots of any two bouquets have no common edge.

(c) For all bouquets $b_{i}$ there exists at least one flower which has no common edge with the root of $b_{j}$ for all $j \neq i$.

Proof. (i) $\Rightarrow$ (ii): It is clear that if (a) or (b) not holds, then $\left[z\left(b_{1}\right)\right] \cdots\left[z\left(b_{l}\right)\right]=0$. Suppose there exists an $i$, such that each flower of $b_{i}$ has common edge with the root of some $b_{j}$. Since $d\left(e_{q_{i}} \wedge e_{q_{i 1}} \wedge \cdots \wedge e_{q_{i p_{i}}}\right)=x_{q_{i}} e_{q_{i 1}} \wedge \cdots \wedge e_{q_{i p_{i}}}-x_{q_{i 1}} e_{q_{i}} \wedge e_{q_{i 2}} \wedge \cdots \wedge$ $e_{q_{i p_{i}}}+\cdots+(-1)^{p_{i}} x_{q_{i p_{i}}} e_{q_{i}} \wedge e_{q_{i 1}} \wedge \cdots \wedge e_{q_{i, p_{i}-1}}$, we have

$$
\left[z\left(b_{i}\right)\right]=\sum_{k=1}^{p_{i}}\left[(-1)^{k+1} x_{q_{i k}} e_{q_{i}} \wedge e_{q_{i 1}} \wedge \cdots \wedge \widehat{e_{q_{i k}}} \wedge \cdots \wedge e_{q_{i p_{i}}}\right] .
$$

Since $x_{q_{i k}}$ has a common edge with the root of some $b_{j}$ for all $k \in\left\{1, \ldots, p_{i}\right\}$, we have $\left[z\left(b_{i}\right)\right]\left[z\left(b_{j}\right)\right]=0$, a contradiction.

(ii) $\Rightarrow$ (i): We prove the assertion by induction on $l$. The case $l=1$ follows from Remark 2.11. Let $\Delta^{\prime}$ be the subforest of $\Delta$ obtained as follows: If one stem of our bouquets is a leaf of $\Delta$, then let $\Delta^{\prime}=\Delta$. Otherwise let $F_{1}$ be any leaf of $\Delta$, and let $\Delta_{1}=\left\langle\mathcal{F}(\Delta) \backslash\left\{F_{1}\right\}\right\rangle$. Notice that $\Delta_{1}$ is again a forest containing all our bouquets. If one stem of our bouquets is a leaf of $\Delta_{1}$, then let $\Delta^{\prime}=\Delta_{1}$. Otherwise let $F_{2}$ be any leaf of $\Delta_{1}$, and let $\Delta_{2}=\left\langle\mathcal{F}\left(\Delta_{1}\right) \backslash\left\{F_{2}\right\}\right\rangle$. Proceeding in this way we obtain a subforest $\Delta^{\prime}$ of $\Delta$ such that

$$
\Delta=\left\langle\mathcal{F}\left(\Delta^{\prime}\right), F_{s}, \ldots, F_{1}\right\rangle,
$$

where $F_{r}$ is a leaf of $\left\langle\mathcal{F}\left(\Delta^{\prime}\right), F_{s}, \ldots, F_{r}\right\rangle$ for $r \in\{1, \ldots, s\}$, and such that some stem of our bouquets, say $\left\{x_{q_{i}}, x_{q_{i k}}\right\}$, is a leaf of $\Delta^{\prime}$. Let $I^{\prime}$ be the edge ideal of $\Delta^{\prime}$, 
$\Gamma=\left\langle\mathcal{F}\left(\Delta^{\prime}\right) \backslash\left\{x_{q_{i}}, x_{q_{i k}}\right\}\right\rangle$ with edge ideal $J^{\prime}$, and let $\Gamma^{\prime}$ be the simplicial complex with facet ideal $J^{\prime}: I^{\prime}$. By Lemma 2.8, $\Gamma^{\prime}$ is a forest.

If $p_{i}>1$, then $x_{q_{i k}}$ must be the free vertex of $\left\{x_{q_{i}}, x_{q_{i k}}\right\}$ in $\Delta^{\prime}$. If $p_{i}=1$, then $b_{i}=\left(x_{q_{i}} ; x_{q_{i 1}}\right)$. It may be that $x_{q_{i 1}}$ is not the free vertex of $\left\{x_{q_{i}}, x_{q_{i 1}}\right\}$ in $\Delta^{\prime}$. Then we replace $b_{i}$ by the bouquet $b_{i}^{\prime}=\left(x_{q_{i 1}} ; x_{q_{i}}\right)$.

Notice that the bouquets $b_{1}, \ldots, b_{i-1}, b_{i}^{\prime}, b_{i+1}, \ldots, b_{l}$ again satisfy all conditions in (ii), and since $\left[b_{i}\right]=\left[b_{i}^{\prime}\right]$ we also have $\left[b_{1}\right] \cdots\left[b_{l}\right]=\left[b_{1}\right] \cdots\left[b_{i-1}\right]\left[b_{i}^{\prime}\right]\left[b_{i+1}\right] \cdots\left[b_{l}\right]$. Therefore we may as well assume that in any case the flower $x_{q_{i k}}$ is the free vertex of $\left\{x_{q_{i}}, x_{q_{i k}}\right\}$ in $\Delta^{\prime}$.

It follows from the definition of $\Gamma^{\prime}$ that all the other flowers of $b_{i}$ are isolated vertices of $\Gamma^{\prime}$. Recall that a vertex in a simplicial complex $\Sigma$ is called isolated if it has no common edge with any other vertex in $\Sigma$.

We distinguish two cases:

Case 1: The root of the bouquet $b_{i}$ has no common edge with any flower in the other bouquets.

In this case $b_{1}, \ldots, b_{i-1}, b_{i+1}, \ldots, b_{l}$ are bouquets in $\Gamma^{\prime}$, and this set of bouquets satisfies all conditions in (ii). By induction hypothesis, $\left[b_{1}\right] \cdots\left[b_{i-1}\right]\left[b_{i+1}\right] \cdots\left[b_{l}\right] \neq 0$ in $H_{.}\left(R /\left(J^{\prime}: I^{\prime}\right)\right)$. Since $x_{q_{i m}}$ is an isolated vertices in $\Gamma^{\prime}$ for $m \in\left\{1, \ldots, p_{i}\right\}$ and $m \neq k$, by Corollary 2.4 , we have $\left[b_{1}\right] \cdots\left[b_{i-1}\right]\left[b_{i+1}\right] \cdots\left[b_{l}\right]\left[e_{q_{i 1}} \wedge \cdots \wedge \widehat{e_{q_{i k}}} \wedge\right.$ $\left.\cdots \wedge e_{q_{i p_{i}}}\right] \neq 0$ in $H_{.}\left(R /\left(J^{\prime}: I^{\prime}\right)\right)$. By Theorem 2.3. for any basis element $[z]$ of $H_{r-1}\left(R /\left(J^{\prime}: I^{\prime}\right)(-2)\right),\left[z \wedge x_{q_{i}} e_{q_{i k}}\right]$ is a basis element of $H_{r}\left(R / I^{\prime}\right)$. Since $z=$ $z\left(b_{1}\right) \cdots \widehat{z\left(b_{i}\right)} \cdots z\left(b_{l}\right)\left(e_{q_{i 1}} \wedge \cdots \wedge \widehat{e_{q_{i k}}} \wedge \cdots \wedge e_{q_{i_{i}}}\right)$ is a cycle in $K .\left(R /\left(J^{\prime}: I^{\prime}\right)(-2)\right)$, it follows that $0 \neq\left[z \wedge x_{q_{i}} e_{q_{i k}}\right]=\left[b_{1}\right] \cdots\left[b_{l}\right]$ in $H .\left(R / I^{\prime}\right)$. By Corollary 2.5, we have $\left[b_{1}\right] \cdots\left[b_{l}\right] \neq 0$ in $H_{.}(R / I)$.

Case 2. There exists an integer $j \neq i$ such that the root $x_{q_{i}}$ has a common edge with some flower of $b_{j}$.

Let $C$ be the set of integers having this property, and let $j \in C$. Since $\Delta$ is a tree, there exists only one flower of $b_{j}$ which has a common edge with $x_{q_{i}}$, because otherwise $\Delta$ would have a cycle. And by the condition (c) in (ii), we have $p_{j}>1$. For $j \in C$, let

$$
b_{j}^{\prime}= \begin{cases}b_{j}, & \text { if } j \notin C, \\ \left(x_{q_{j}} ; x_{q_{j 1}}, \ldots, \widehat{x_{q_{j k}}}, \ldots, x_{q_{j p_{j}}}\right), & \text { if } j \in C \text { and }\left\{x_{q_{i}}, x_{q_{j k}}\right\} \text { is an edge. }\end{cases}
$$

Then $b_{1}^{\prime}, \ldots, b_{i-1}^{\prime}, b_{i+1}^{\prime}, \ldots, b_{l}^{\prime}$ are bouquets of $\Gamma^{\prime}$, and this set of bouquets satisfies all the conditions in (ii). For all $j \in C$, let $\left\{x_{q_{i}}, x_{q_{j_{k}}}\right\}$ be the unique common edge of the root $x_{q_{i}}$ of $b_{i}$ with the flower $x_{q_{j_{k}}}$ in $b_{j}$. Then $x_{q_{j_{k}}}$ is an isolated vertex of $\Gamma^{\prime}$. Hence in $\Gamma^{\prime}$ we are in the same situation as in Case 1, and so as before the result follows by induction.

Definition 2.15. Let $\Delta$ be a simple graph, that is, for each edge $\left\{x_{i}, x_{j}\right\}$ of $\Delta$, $x_{i} \neq x_{j}$. Two edges $\left\{x_{i}, x_{j}\right\}$ and $\left\{x_{k}, x_{l}\right\}$ are called disconnected if

(a) $\left\{x_{i}, x_{j}\right\} \cap\left\{x_{k}, x_{l}\right\}=\emptyset$;

(b) $\left\{x_{i}, x_{k}\right\},\left\{x_{i}, x_{l}\right\},\left\{x_{j}, x_{k}\right\},\left\{x_{j}, x_{l}\right\}$ are not edges of $\Delta$. 
Corollary 2.16. Let $\Delta$ be a 1-dimensional forest with edge ideal $I$, and $\left\{x_{i_{1}}, x_{j_{1}}\right\}$, $\ldots,\left\{x_{i_{m}}, x_{j_{m}}\right\}$ edges of $\Delta$. Then the following are equivalent:

(i) $\left[x_{i_{1}} e_{j_{1}}\right] \cdots\left[x_{i_{m}} e_{j_{m}}\right] \neq 0$.

(ii) The edges $\left\{x_{i_{1}}, x_{j_{1}}\right\}, \ldots,\left\{x_{i_{m}}, x_{j_{m}}\right\}$ are pairwise disconnected.

Proof. Let $b_{l}=\left\{x_{i_{l}} ; x_{j_{l}}\right\}, l=1, \ldots, m$. Then $b_{l}$ is a bouquet with one flower. Notice that $b_{l}^{\prime}=\left\{x_{j_{l}} ; x_{i_{l}}\right\}$ is also a bouquet with one flower of $\Delta$. Since $\left[z\left(b_{l}\right)\right]=\left[z\left(b_{l}^{\prime}\right)\right]$, we have $\left[z\left(b_{1}\right)\right] \cdots\left[z\left(b_{m}\right)\right] \neq 0$ if and only if $\left[z\left(b_{1}\right)\right] \cdots\left[z\left(b_{l-1}\right)\right]\left[z\left(b_{l}^{\prime}\right)\right]\left[z\left(b_{l+1}\right)\right]\left[z\left(b_{m}\right)\right] \neq 0$. Hence we may choose $x_{i_{l}}$ or $x_{j_{l}}$ as the root of $b_{l}$.

(i) $\Rightarrow$ (ii): If $\left[x_{i_{1}} e_{j_{1}}\right] \cdots\left[x_{i_{m}} e_{j_{m}}\right] \neq 0$, then all conditions in (ii) of Proposition 2.14 hold. Hence all vertices occurring in these edges are pairwise distinct, and $\left\{x_{i_{l}}, x_{j_{l}}\right\}, l=1, \ldots, m$ are the only edges in the subgraph of $\Delta$ restricted to the vertices $\left\{x_{i_{1}}, \ldots, x_{i_{m}}, x_{j_{1}} \ldots, x_{j_{m}}\right\}$. It follows that $\left\{x_{i_{1}}, x_{j_{1}}\right\}, \ldots,\left\{x_{i_{m}}, x_{j_{m}}\right\}$ are pairwise disconnected.

(ii) $\Rightarrow$ (i): If $\left\{x_{i_{1}}, x_{j_{1}}\right\}, \ldots,\left\{x_{i_{m}}, x_{j_{m}}\right\}$ are pairwise disconnected, then the set of bouquets $b_{1}, \ldots, b_{m}$ satisfies all conditions in (ii) of Proposition [2.14. Hence $\left[x_{i_{1}} e_{j_{1}}\right] \cdots\left[x_{i_{m}} e_{j_{m}}\right] \neq 0$.

Moreover, we have

Corollary 2.17. Let $\Delta$ be a 1-dimensional tree, and $b_{1}, \ldots, b_{l}$ bouquets of $\Delta$. If the set of these bouquets satisfies the condition (ii) of Proposition 2.14, then there exists one stem in each bouquet, such that these stems are pairwise disconnected.

Proof. We refer to the notation in the proof of Proposition 2.14. By the proof 2.14 (ii) $\Rightarrow$ (i) in each step we get a leaf $\left\{x_{p_{i}}, x_{p_{i k}}\right\}$ in the subforest of the previous one. The arguments in the proof show that these stems are pairwise disconnected.

By using Proposition 2.14, Corollary 2.16] and Corollray 2.17] we conclude:

Theorem 2.18. Let $\Delta$ be a 1-dimensional forest, I its edge ideal. Then the regularity of $R / I$ is the maximal number $j$, for which there exist $j$ edges which are pairwise disconnected.

Remark 2.19. In Theorem 2.18, the assumption that $\Delta$ is a forest is important. If $\Delta$ has a cycle, then the assertion might not be true.

For example, let $\Delta$ be a graph with edge ideal $I=(a b, b c, c d, d e, e a)$. Then the regularity of $R / I$ is 2 , but the maximal number of the pairwise disconnected edges in $\Delta$ is 1 .

\section{LiNEAR TREES}

In general, it is not easy to determine the Betti numbers of an $R$-module $M$, but for a facet ideal $I$ of a pure tree which is connected in codimension 1 , we can describe the linear part of the resolution of $R / I$.

We know that if $M$ is a graded $R$-module, $z \in R$ is a homogeneous element of degree 1 , and $z$ is a non-zero divisor of $M$, then

$$
b_{i j}(M / z M)=b_{i j}(M)+b_{i-1, j}(M(-1))=b_{i j}(M)+b_{i-1, j-1}(M) .
$$


In fact, if $F$. is a graded minimal free resolution of $M$, then the mapping cone of $F \cdot(-1) \stackrel{z}{\longrightarrow} F$. is the minimal graded free resolution of $M / z M$.

Lemma 3.1. Let $L$ be a monomial ideal in $R$ with $G(L)=\left\{g_{1}, \ldots, g_{l}\right\}$. Suppose that $\operatorname{deg}\left(g_{r}\right)=1$ for $r=1, \ldots, s$, and $\operatorname{deg}\left(g_{r}\right)>1$ for $r=s+1, \ldots, l$. Then $b_{i i}(R / L)=\left(\begin{array}{l}s \\ i\end{array}\right)$.

Proof. We may assume that $g_{i}=x_{i}$ for $i \in[s]$. Then for all $i \in[s], x_{i}$ does not divide any $g_{j}$ for $j>s$, because $\left\{g_{1}, \ldots, g_{l}\right\}$ is a minimal set of generators of $L$. Hence $g_{1}, \ldots, g_{s}$ is a regular sequence modulo $\left(g_{s+1}, \ldots, g_{l}\right)$. Hence the assertion follows by induction on $s$ from (2).

Definition 3.2. Let $\Delta$ be a $d$-dimensional pure tree connected in codimension 1 and $G$ a face of dimension $d-1$. If $G$ is contained in at least two facets of $\Delta$, then we call $G$ an adjacent face.

Proposition 3.3. Let $\Delta$ be a d-dimensional pure tree with $m$ facets, I its facet ideal. Suppose $\Delta$ is connected in codimension 1. For each adjacent face $G \in \Delta$, let $m(G)=|\{F \in \mathcal{F}(\Delta): G \subset F\}|$. Then

$$
b_{i, i+d}(R / I)= \begin{cases}m, & \text { if } i=1, \\
\sum_{G}\left(\begin{array}{c}
m(G) \\
i
\end{array}\right), & \text { if } i \geq 2 .\end{cases}
$$

Proof. Let $\Delta=\left\langle F_{1}, \ldots, F_{m}\right\rangle$ such that $F_{1}, \ldots, F_{m}$ is a leaf order. We prove the proposition by induction on $m$. The case $m=1$ is trivial. Let $\Gamma=\left\langle F_{1}, \ldots, F_{m-1}\right\rangle$ and $J$ be the facet ideal of $\Gamma$. By Corollary 2.6 and Lemma 3.1, we know

$$
\begin{aligned}
b_{i, i+d}(R / I) & =b_{i, i+d}(R / J)+b_{i-1, i+d}(R /(J: I)(-(d+1))) \\
& =b_{i, i+d}(R / J)+b_{i-1, i-1}(R /(J: I)),
\end{aligned}
$$

and

$$
b_{i-1, i-1}(R /(J: I))=\left(\begin{array}{c}
s \\
i-1
\end{array}\right),
$$

where $s=\left|\left\{F_{j}: \operatorname{dim}\left(F_{j} \cap F_{m}\right)=d-1, \quad j=1, \ldots, m-1\right\}\right|$, because $x_{i} \in J: I$ if and only if $x_{i} \in F_{j} \backslash F_{m}$ for some $F_{j}$ in this set.

Let $G$ be an adjacent face of $\Gamma$ and $m^{\prime}(G)=|\{F \in \mathcal{F}(\Gamma): G \subset F\}|$. By our induction hypothesis

$$
b_{i, i+d}(R / J)= \begin{cases}m-1, & \text { if } i=1 \\
\sum_{G}\left(\begin{array}{c}
m^{\prime}(G) \\
i
\end{array}\right), & \text { if } i \geq 2 .\end{cases}
$$

So

$$
b_{i, i+d}(R / I)= \begin{cases}m-1+1=m, & \text { if } i=1 \\
\sum_{G}\left(\begin{array}{c}
m^{\prime}(G) \\
i
\end{array}\right)+\left(\begin{array}{c}
s \\
i-1
\end{array}\right)=\sum_{G}\left(\begin{array}{c}
m(G) \\
i
\end{array}\right), & \text { if } i \geq 2\end{cases}
$$

For a $d$-dimensional pure tree $\Delta$, we assign to each face $G$ with dimension $d-1$ an degree, namely

$$
\operatorname{deg}(G)=\mid\{F: F \text { is a facet of } \Delta \text {, such that } G \subset F\} \mid .
$$


By Proposition 3.3. $b_{i, i+d}(R / I)=\sum_{G}\left(\begin{array}{c}\operatorname{deg}(G) \\ i\end{array}\right)$ for $i \geq 2$, where $I$ is the facet ideal of $\Delta$. (Notice that if $G$ is not an adjacent face, then $\left(\begin{array}{c}\operatorname{deg}(G) \\ i\end{array}\right)=0$ for $i \geq 2$.) If $d=1$, then the face of dimension $d-1$ is just a vertex.

Remark 3.4. Let $\Delta$ be a 1-dimensional tree. Then $b_{2,3}=\sum_{v}\left(\begin{array}{c}\operatorname{deg}(v) \\ 2\end{array}\right)$, where $v$ runs through all the vertices of $\Delta$. In [2], Eliahou and Villarreal proved that for any graph $G, b_{2,3}=|E(L(G))|-N_{t}$, where $N_{t}$ is the number of triangles of $G$ and $L(G)$ is the line graph of $G$. In the case $G$ is a tree, $N_{t}=0$, and $|E(L(G))|$ is just $\sum_{v}\left(\begin{array}{c}\operatorname{deg}(v) \\ 2\end{array}\right)$.

Lemma 3.5. Let $\Delta$ be a d-dimensional pure tree and connected in codimension $1, V$ the set of faces of dimension $d-1$, and $O=\sum_{G \in V} \operatorname{deg}(G)$. Then we have $|\mathcal{F}(\Delta)|-1=O-|V|$.

Proof. The lemma follows by induction on the number of facets, observing that when we add a leaf to the tree, $O$ will increase by $d+1$, and $|V|$ by $d$.

For a $d$-dimensional pure tree $\Delta$ which is connected in codimension 1 , let $b_{0}^{\prime}=|V|$, $b_{1}^{\prime}=O$, and $b_{i}^{\prime}=b_{i, i+d}$ for $i \geq 2$. By using the well-known binomial formula $\sum_{i=0}^{n}(-1)^{i}\left(\begin{array}{c}n \\ i\end{array}\right)=0$, one sees that $\sum_{i}(-1)^{i} b_{i}^{\prime}=0$. Hence together with Lemma 3.5 we have

Proposition 3.6. Let $\Delta$ be a d-dimensional pure tree with the facet ideal I. Suppose $\Delta$ is connected in codimension 1 . Then

$$
1+\sum_{i>0}(-1)^{i} b_{i, i+d}=0 .
$$

In the next section, we will have another property on the Betti numbers of facet ideals which generalizes this proposition.

Definition 3.7. Let $I$ be a monomial ideal in $R$. We say $I$ is a linear quotient ideal, if for some order $f_{1}, \ldots, f_{m}$ of the elements in $G(I)$ the colon ideal $\left(f_{1}, \ldots, f_{i-1}\right): f_{i}$ is generated by monomials of degree 1 for each $i \in[m]$, and all $f_{i}$ have the same degree.

Definition 3.8. Let $\Delta$ be a tree. If its facet ideal $I$ is a linear quotient ideal, then we call $\Delta$ a linear quotient tree. If $I$ has linear resolution, then we call $\Delta$ a linear tree.

Proposition 3.9. Let $\Delta$ be a tree, I its facet ideal.

(i) The following statements are equivalent:

(a) $\Delta$ is a linear quotient tree.

(b) $\Delta$ is a linear tree.

(ii) If $\Delta$ satisfies the equivalent conditions in (i), then $\Delta$ is pure and connected in codimension 1 .

Proof. (i) $(a) \Rightarrow(b)$ : By definition, we have $\Delta$ is pure. Let $d-1$ be the dimension of $\Delta$. We prove the assertion by induction on the number of facets $m$. 
The case $m=1$ is trivial. Suppose $m>1$. Let $F_{1}, \ldots, F_{m}$ be the facets of $\Delta$ and $J=\left(f_{1}, \ldots, f_{m-1}\right)$ such that $J: I$ can be generated by monomials of degree 1 . By induction hypothesis, $R / J$ has a linear resolution. Hence

$$
\operatorname{Tor}_{i}^{R}(K, R / J)_{j}=0 \text { for } j \neq i+d \text { and all } i>0 .
$$

Since $J: I$ is generated by monomials of degree 1 , we have

$$
\operatorname{Tor}_{i}^{R}(K, J: I)_{j}=0 \text { for } j \neq i+1 \text { and all } i>0 .
$$

From the exact sequence

$$
0 \longrightarrow R /(J: I)(-d) \longrightarrow R / J \longrightarrow R / I \longrightarrow 0,
$$

we have the long exact sequence

$$
\begin{aligned}
\cdots & \longrightarrow \operatorname{Tor}_{i}^{R}(K, R /(J: I)(-d)) \longrightarrow \operatorname{Tor}_{i}^{R}(K, R / J) \longrightarrow \operatorname{Tor}_{i}^{R}(K, R / I) \\
& \longrightarrow \operatorname{Tor}_{i-1}^{R}(K, R /(J: I)(-d)) \longrightarrow \quad \cdots,
\end{aligned}
$$

By using (3i) and (44), this long exact sequence implies that $\operatorname{Tor}_{i}^{R}(K, R / I)_{j}=0$ for $j \neq i+d$ and all $i>0$, so $I$ has linear resolution, i.e. $\Delta$ is a linear tree.

$(b) \Rightarrow(a)$ : It is clear that if $\Delta$ is not pure, then $I$ has no linear resolution. We may assume $\Delta$ is a pure tree of dimension $d-1$. Suppose $I$ is not a linear quotient ideal. Let $F_{1}, \ldots, F_{m}$ be a leaf order. Then $L=\left(f_{1}, \ldots, f_{k-1}\right): f_{k}$ is not generated by monomials of degree 1 for some $k \in\{1, \ldots, m\}$, and hence $b_{1,1+j}(R / L) \neq 0$ for some $j>1$. Let $I^{\prime}=\left(f_{1}, \ldots, f_{k}\right)$ and $J^{\prime}=\left(f_{1}, \ldots, f_{k-1}\right)$. By Theorem 2.3 we have the exact sequence

$0 \longrightarrow \operatorname{Tor}_{2}^{R}\left(K, R / J^{\prime}\right) \longrightarrow \operatorname{Tor}_{2}^{R}\left(K, R / I^{\prime}\right) \longrightarrow \operatorname{Tor}_{1}^{R}(K, R / L(-d)) \longrightarrow 0$, which implies that $b_{2,2+j+d}\left(R / I^{\prime}\right) \neq 0$, so $I^{\prime}$ has no linear resolution since $I^{\prime}$ is generated in degree $d$. By Corollary 2.5, $I$ has no linear resolution, a contradiction.

(ii) It is clear that $\Delta$ must be pure. Let $F_{1}, \ldots, F_{m}$ be the facets of $\Delta$ such that $\left(f_{1}, \ldots, f_{k-1}\right): f_{k}$ is generated by monomials of degree 1 for $k=1, \ldots, m$. We prove that $\Delta$ is connected in codimension 1 by induction on $m$. The case $m=1$ is trivial. Assume $m>1$, since $\left(f_{1}, \ldots, f_{m-1}\right)$ is a linear quotient ideal, by induction hypothesis, $\left\langle F_{1}, \ldots, F_{m-1}\right\rangle$ is connected in codimension 1 . To show $\Delta$ is connected in codimension 1 , we only need to show that for any facet $F_{i}$, with $i<m$, there exists a proper chain between $F_{i}$ and $F_{m}$. Since $\left(f_{1}, \ldots, f_{m-1}\right): f_{m}$ is generated by monomials of degree 1 , we have that all the facets of $\left\langle F_{m}\right\rangle \cap\left\langle F_{1}, \ldots, F_{m-1}\right\rangle$ are of dimension $d-1$. Hence there exists an integer $j<m$ such that $\operatorname{dim}\left(F_{j} \cap F_{m}\right)=d-1$. Since $F_{i}$ and $F_{j}$ both are facets of the tree $\left\langle F_{1}, \ldots, F_{m-1}\right\rangle$, there exists a proper chain $F_{i}=F_{i_{0}}, \ldots, F_{i_{l}}=F_{j}$ between $F_{i}$ and $F_{j}$. Hence $F_{i}=F_{i_{0}}, \ldots, F_{i_{l}}=F_{j}, F_{m}$ is a proper chain between $F_{i}$ and $F_{m}$.

By Proposition 3.3 and Proposition 3.9 the Betti numbers of a linear tree can now be described as follows:

Corollary 3.10. Let $\Delta$ be a d-dimensional linear tree with $m$ facets, $I$ its facet ideal. Then

$$
b_{i}(R / I)= \begin{cases}m, & \text { if } i=1, \\
\sum_{G}\left(\begin{array}{c}
m(G) \\
i
\end{array}\right), & \text { if } i \geq 2,\end{cases}
$$


where the sum is taken over all $(d-1)$-dimensional faces $G$ of $\Delta$, and $m(G)=$ $|\{F \in \mathcal{F}(\Delta): G \subset F\}|$.

Later in this section, we will classify all linear trees of a given dimension. For this, we need some preparation.

Lemma 3.11. Let $\Delta$ be a linear tree, $\Gamma$ a subcomplex of $\Delta$ which is connected in codimension 1 . Then $\Gamma$ is a linear tree.

Proof. It is clear that $\Gamma$ is again a pure tree. We may assume $\Gamma \neq \Delta$. We claim there exists an order of the facets $F_{1}, \ldots, F_{l}$ of $\langle\mathcal{F}(\Delta) \backslash \mathcal{F}(\Gamma)\rangle$ such that $\left\langle\mathcal{F}(\Gamma), F_{1}, \ldots, F_{i}\right\rangle$ is connected in codimension $1, i=1 \ldots, l$. In fact, let $F \in \mathcal{F}(\Gamma)$ and $G \in \mathcal{F}(\Delta) \backslash \mathcal{F}(\Gamma)$ be any two facets. Since $\Delta$ is connected in codimension 1 , there exists a unique irredundant proper chain from $F$ to $G$. Let $F_{1}$ be the first facet in this chain which does not belong to $\Gamma$. Then it is obvious that $\left\langle\mathcal{F}(\Gamma), F_{1}\right\rangle$ is connected in codimension 1. The claim follows by induction on $|\mathcal{F}(\Delta) \backslash \mathcal{F}(\Gamma)|$.

By Corollary 1.15, $F_{i}$ is a leaf of $\left\langle\mathcal{F}(\Gamma), F_{1}, \ldots, F_{i}\right\rangle$ for $i=1, \ldots, l$. Let $I$ and $J$ be the facet ideals of $\Delta$ and $\Gamma$, respectively. By Corollary 2.5 , $b_{i, i+j}(J) \leq b_{i, i+j}(I)$ for any $i$ and $j$. Since $I$ has linear resolution, this implies that $J$ has linear resolution.

Lemma 3.12. Let $\Delta$ be a linear tree, $F$ and $G$ any two facets of $\Delta$. Let $F=$ $F_{0}, \ldots, F_{m}=G$ be the irredundant proper chain between $F$ and $G$. Then $\left(f_{0}, \ldots\right.$, $\left.f_{l-1}\right): f_{l}$ is generated by monomials of degree $1, l=0, \ldots, m$.

Proof. Since $F_{0}, \ldots, F_{m}$ is an irredundant proper chain, $\left\langle F_{0}, \ldots, F_{i}\right\rangle$ is a linear tree for all $i$, see Lemma 3.11. Assume there exists an $l$ such that $\left(f_{0}, \ldots, f_{l-1}\right): f_{l}$ is not generated by monomials of degree 1 . Since $F_{l}$ is a leaf of $\left\langle F_{0}, \ldots, F_{l}\right\rangle$, it follows from Theorem 2.3 that $\left\langle F_{0}, \ldots, F_{l}\right\rangle$ is not a linear tree, a contradiction.

Proposition 3.13. Let $\Delta$ be a pure tree of dimension $d, F$ and $G$ any two facets with $\operatorname{dim}(F \cap G)=d-k$, for some $k \in[d+1]$. Then

(i) $\operatorname{dist}(F, G) \geq k$;

(ii) $\operatorname{dist}(F, G)=k$, if $\Delta$ is a linear tree.

Proof. (i) is obvious. Now let $\Delta$ be a linear tree, and suppose that $\operatorname{dist}(F, G)>k$. Let $F=F_{0}, \ldots, F_{l}=G$ be the irredundant proper chain between $F$ and $G$, where $l>k$. Let $H=F \cap G$. By Proposition 1.12, $H \subset F_{k}$ for $k=0, \ldots, l$.

Let $\left\{x_{i}\right\}=F_{i} \backslash F_{i+1}$ for $i=0, \ldots, l-1$. We claim that $\left\{x_{0}, \ldots, x_{l-1}\right\} \subset F_{0}$, and that the elements $x_{i}$ are pairwise distinct.

Assume $x_{j} \notin F_{0}$ for some $j=0, \ldots, l-1$. Since $F_{0}, \ldots, F_{j}$ is an irredundant proper chain, it follows that $F_{k} \cap F_{j+1}$ is a proper subset of $F_{j} \cap F_{j+1}$ for $k<j$. This implies that $\left|F_{k} \backslash F_{j+1}\right|>1$ for all $k<j$, while $F_{j} \backslash F_{j+1}=\left\{x_{j}\right\}$. On the other hand, $\left(f_{0}, \ldots, f_{j}\right): f_{j+1}$ is generated by monomials of degree 1 . This implies that $x_{j} \in F_{k}$ for all $k \leq j$. In particular, $x_{j} \in F_{0}$, a contradiction. Since $F_{i}, \ldots, F_{l}$ is an irredundant proper chain, $F_{i}$ is a leaf of $\left\langle F_{i}, \ldots, F_{l}\right\rangle$ for all $i \in\{0, \ldots, l-1\}$. Hence $x_{i} \notin F_{k}$ for all $k>i$. So the $x_{i}$ are pairwise distinct, and $x_{i} \notin H$ for $i=0, \ldots, l-1$.

So we have $H \cup\left\{x_{0}, \ldots, x_{l-1}\right\} \subseteq F_{0}$. Hence $\left|F_{0}\right| \geq d-k+1+l>d+1$, a contradiction. 
Definition 3.14. Let $\Delta$ be a $d$-dimensional pure tree and connected in codimension 1. If for any two facets $F$ and $G$ with $\operatorname{dim}(F \cap G)=d-k, k=1, \ldots, d+1$, we have $\operatorname{dist}(F, G)=k$, then we say $\Delta$ has the intersection property.

Remark 3.15. Let $\Delta$ be a $d$-dimensional tree with intersection property, and $l$ the diameter of $\Delta$. Then

(i) $l \leq d+1$, and

(ii) for any irredundant proper chain $\mathcal{C}$ in $\Delta$, and any face $H$ in $\Gamma$ of dimension $d-k$, where $\Gamma$ is the simplicial complex generated by $\mathcal{C}$, one has that $H$ is contained in at most $k+1$ facets of $\Gamma$.

In fact, it is clear that for any two facets $F$ and $G$ of $\Delta$, $\operatorname{dist}(F, G) \leq d+1$. Hence $l \leq d+1$.

Assume $H$ is contained in more than $k+1$ facets of $\Gamma$. Since $\Gamma$ is generated by the irredundant proper chain $\mathcal{C}$, there exist two facets $F$ and $G$ of $\Gamma$ such that $H \subseteq F \cap G$ and $\operatorname{dist}(F, G)>k$. But $\operatorname{dim}(F \cap G) \geq \operatorname{dim} H=d-k$, contradicting Proposition 3.13 .

Proposition 3.16. Let $\Delta$ be a linear tree of dimension d, and $G$ an adjacent face. Let $\Gamma=\langle\mathcal{F}(\Delta), F\rangle$, where $F$ is a facet of dimension d and $\langle F\rangle \cap \Delta=\langle G\rangle$. Then $\Gamma$ is a linear tree.

Proof. By Proposition 3.9. we have $\Delta=\left\langle F_{1}, \ldots, F_{m}\right\rangle$ such that $\left(f_{1}, \ldots, f_{i-1}\right): f_{i}$ is generated by monomials of degree $1, i=1, \ldots, m$. Let $F_{i_{1}}, \ldots, F_{i_{l}}$ be all the facets of $\Delta$ which contains $G$, and $x_{i_{j}}=F_{i_{j}} \backslash F$ for $j=1, \ldots, l$, where $l>1$. We prove that $\left(f_{1}, \ldots, f_{m}\right): f=\left(x_{i_{1}}, \ldots, x_{i_{l}}\right)$ (which implies that $\Gamma$ is also a linear tree).

It is clear that $\left(x_{i_{1}}, \ldots, x_{i_{l}}\right) \subseteq\left(f_{1}, \ldots, f_{m}\right): f$. In order to prove the converse inclusion, we first notice that there exists no facet $F_{p}$ of $\Delta$, such that $F_{p} \cap F_{i_{j}}=\emptyset$ for all $j=1, \ldots, l$. Otherwise by Proposition [3.13, $\operatorname{dist}\left(F_{i_{j}}, F_{p}\right)=d+1$ for all $j=1, \ldots, l$. Since $l>1$, this contradicts Lemma 1.17

It remains to show that for any facet $F_{p}$ of $\Delta$ we have $F_{p} \cap\left\{x_{i_{1}}, \ldots, x_{i_{l}}\right\} \neq \emptyset$. Suppose there exists a facet $F_{p}$ such that $F_{p} \cap\left\{x_{i_{1}}, \ldots, x_{i_{l}}\right\}=\emptyset$, then $p \neq i_{j}$, and hence we have $F_{p} \cap G=F_{p} \cap F_{i_{j}} \neq \emptyset$ for $j=1, \ldots, l$. Let $\operatorname{dim}\left(F_{p} \cap F_{i_{j}}\right)=d-k$. Then by Proposition 3.13, $\operatorname{dist}\left(F_{p}, F_{i_{j}}\right)=k$ for $j=1, \ldots, l$. Again, since $l>1$, this contradicts Lemma 1.17.

Now we can show

Theorem 3.17. Let $\Delta$ be a tree. Then the following are equivalent:

(i) $\Delta$ is a linear tree.

(ii) $\Delta$ has intersection property.

Proof. (i) $\Rightarrow$ (ii) follows from Proposition 3.13 .

(ii) $\Rightarrow$ (i): We prove the assertion by induction on the number of facets $m$ of $\Delta$. The case $m=1$ is trivial. Assume $m>1$. Let $F$ be a leaf of $\Delta$. By induction hypothesis, $\langle\mathcal{F}(\Delta) \backslash\{F\}\rangle$ is a linear tree because it still satisfies the intersection property. Let $H=\langle F\rangle \cap\langle\mathcal{F}(\Delta) \backslash\{F\}\rangle$; if $\left|\mathcal{U}_{\Delta}(F)\right|>1$, then $H$ is an adjacent face of $\langle\mathcal{F}(\Delta) \backslash\{F\}\rangle$. Hence $\Delta$ is a linear tree by Proposition 3.16. If $\left|\mathcal{U}_{\Delta}(F)\right|=1$, let $\left\{F^{\prime}\right\}=\mathcal{U}_{\Delta}(F)$ and $\{x\}=F^{\prime} \backslash F$. 
We claim $x$ is contained in any facet of $\langle\mathcal{F}(\Delta) \backslash\{F\}\rangle$. Hence, since $\langle\mathcal{F}(\Delta) \backslash\{F\}\rangle$ is a linear tree, $\Delta$ is a linear tree, too.

In order to prove the claim, consider $G \in \mathcal{F}(\Delta), G \neq F$, and let $E=F \cap G$ and assume that $\operatorname{dim} E=d-k$. Then $G=\left\{x_{1}, \ldots, x_{k}\right\} \cup E$ and $F=\left\{y_{1}, \ldots, y_{k}\right\} \cup E$, where all the elements in $\left\{x_{1}, \ldots, x_{k}, y_{1}, \ldots, y_{k}\right\}$ are pairwise distinct. Since $\Delta$ has intersection property, $\Delta$ is pure and connected in codimension 1 , and $\operatorname{dist}(F, G)=k$. Hence there exists an irredundant proper chain $G=F_{0}, F_{1}, \ldots F_{k}=F$ between $G$ and $F$. Since $F$ is a leaf of $\Delta$ and $\left\{F^{\prime}\right\}=\mathcal{U}_{\Delta}(F)$, we have $F_{k-1}=F^{\prime}$. Since $\left|F_{i} \backslash F_{i+1}\right|=1$ for all $i$, we may assume $F_{i}=\left\{y_{1}, \ldots, y_{i}, x_{i+1}, \ldots, x_{k}\right\} \cup E$ for $i=1, \ldots, k$. Hence $F_{k-1}=\left\{y_{1}, \ldots, y_{k-1}, x_{k}\right\} \cup E$. But on the other hand, $F_{k-1}=$ $F^{\prime}=\left\{y_{1}, \ldots, y_{k-1}, x\right\} \cup E$. Hence $x=x_{k} \in G$.

Remark 3.18. In the case that $\Delta$ is a 1-dimensional tree, the intersection property is equivalent to the condition that the distance between any two edges in $\Delta$ is at most 2 , and this is equivalent to say that the complement $\bar{\Delta}$ of the graph $\Delta$ is a triangulated graph. This coincides with the result of Fröberg in [5] .

\section{The Alternating SUm PROPERTy OF FACET IDEAlS}

In this section we show that for a special class of facet ideals $I$ the Betti numbers have the property that $\sum_{i}(-1)^{i} b_{i, i+j}(R / I)=0$ for all $j>d$, where $d$ is the least degree of the generators. These class of ideals include facet ideals of trees (not necessary pure) which are connected in codimension 1.

Definition 4.1. Let $I$ be a monomial ideal in $R$ with $G(I)=\left\{f_{1} \ldots, f_{m}\right\}$ and $d=\min \left\{\operatorname{deg}\left(f_{i}\right): i=1, \ldots, m\right\}$. We say that $I$ has the alternating sum property, if

$$
\sum_{i \geq 1}(-1)^{i} b_{i, i+j}(R / I)= \begin{cases}-1, & \text { for } j=d \\ 0, & \text { for } j>d\end{cases}
$$

To proof the main theorem of this section, we need the following fact:

Lemma 4.2. Let I be a monomial ideal in $R$. Suppose $G(I)$ contains a monomial of degree 1 . Then $\sum_{i}(-1)^{i} b_{i, i+j}(R / I)=0$ for all $j$.

Proof. Let $G(I)=\left\{m_{1}, \ldots, m_{l}, x\right\}, J=\left(m_{1}, \ldots, m_{l}\right)$. Then $x$ does not divide $m_{j}$ for $j=1, \ldots, l$, and $J: I=J$. By Theorem 2.3 we have for $i>0$

$$
\begin{aligned}
b_{i, i+j}(R / I) & =b_{i, i+j}(R / J)+b_{i-1, i+j}(R /(J: I)(-1)) \\
& =b_{i, i+j}(R / J)+b_{i-1, i-1+j}(R /(J: I)) \\
& =b_{i, i+j}(R / J)+b_{i-1, i-1+j}(R / J) .
\end{aligned}
$$

From this it follows that $\sum_{i}(-1)^{i} b_{i, i+j}(R / I)=0$.

Remark 4.3. With the same arguments as in the proof of Lemma4.2 one can show more generally: Let $J$ be a graded ideal in $R, I=(J, f)$, where $\operatorname{deg}(f)=1$. If $f$ is regular on $R / J$, then $\sum_{i}(-1)^{i} b_{i, i+j}(R / I)=0$ for all $j$. 
Proposition 4.4. Let $\Delta$ be a simplicial complex with facet ideal $I$. If there exists an order of the facets $F_{1}, \ldots, F_{m}$ of $\Delta$ such that for each $i=2, \ldots, m, F_{i} \backslash \bigcup_{j<i} F_{j} \neq \emptyset$, and there exists $j<i$ such that $\left|F_{j} \backslash F_{i}\right|=1$. Then $I$ has the alternating sum property.

Proof. We prove this proposition by induction on $m$. The case $m=1$ is trivial. Let $d=\min \left\{\operatorname{deg}\left(f_{i}\right): i=1, \ldots, m-1\right\}, d^{\prime}=\operatorname{deg}\left(f_{m}\right)$, and $J=\left(f_{1}, \ldots, f_{m-1}\right)$. Since $\left|F_{j} \backslash F_{m}\right|=1$ for some $j<m$ it follows that $d^{\prime} \geq d$, and that $G(J: I)$ contains at least one monomial of degree 1. By Lemma 4.2.

$$
\sum_{i}(-1)^{i} b_{i, i+j}(R /(J: I))=0 \text { for any } j .
$$

On the other hand by Theorem 2.3. we have

$$
b_{i, i+j}(R / I)=b_{i, i+j}(R / J)+b_{i-1, i+j-d^{\prime}}(R /(J: I)),
$$

for $i>0$, since $F_{m} \backslash \bigcup_{j<m} F_{j} \neq \emptyset$. By induction hypothesis $J$ has the alternating sum property. Hence one sees that $I$ has the alternating sum property by using (5) and (6).

Corollary 4.5. Let $\Delta$ be a pure quasi-tree connected in codimension 1 with facet ideal I. Then I has the alternating sum property.

Proof. Since $\Delta$ is a quasi-tree, there exists a leaf order of facets $F_{1}, \ldots, F_{m}$. The assertion follows from Proposition 4.4 immediately.

The next result shows that in Corollary 4.5 we can skip the assumption that $\Delta$ is pure if we assume that $\Delta$ is a tree.

Theorem 4.6. Let $\Delta$ be a tree connected in codimension 1 with facet ideal I. Then $I$ has the alternating sum property.

Proof. We prove the assertion by induction on the number of facets $m$. The case $m=1$ is trivial. Assume $m>1$. Let $d=\operatorname{dim} \Delta$. There are two cases.

Case 1. There exists only one facet $F$ of dimension $d$. Then $F$ must be a leaf. Otherwise, there exist two facets $G_{1}, G_{2}$ such that $F \cap G_{1} \nsubseteq F \cap G_{2}$ and $F \cap G_{2} \nsubseteq$ $F \cap G_{1}$. Since $\Delta$ is connected in codimension 1 and $\operatorname{dim} G_{i}<d, i=1,2$, there exists a chain $\mathcal{C}$ between $G_{1}$ and $G_{2}$ which does not include $F$. Then the simplicial subcomplex $\Gamma$ whose facets are the elements of $\mathcal{C}$ and $F$ has no leaf, a contradiction.

We choose a $G \in \mathcal{U}_{\Delta}(F)$ (see Definition 1.8) of maximal dimension. Since $\Delta$ is connected in codimension 1, we have $\operatorname{dim} G=\operatorname{dim}\langle\mathcal{F}(\Delta) \backslash\{F\}\rangle$ and $\operatorname{dim}(F \cap G)=$ $\operatorname{dim} G-1$, i.e. $|G \backslash F|=1$. Since $F$ is a leaf, $\langle\mathcal{F}(\Delta) \backslash\{F\}\rangle$ is a tree with $m-1$ facets which is connected in codimension 1. By induction hypothesis there exists a leaf order of facets $F_{1}, \ldots, F_{m-1}$ such that for each $i=2, \ldots, m-1, F_{i} \backslash \bigcup_{j<i} F_{j} \neq \emptyset$, and there exists $j<i$ such that $\left|F_{j} \backslash F_{i}\right|=1$. Let $F=F_{m}$. We see that $F_{1}, \ldots, F_{m}$ satisfy the conditions of Theorem 4.4 in this order.

Case 2. There exist more than one facets of dimension $d$. Let $G_{1}, \ldots, G_{s}$ be all of these facets, where $s>1$. Then for any $i$ and $j$, the facets in any proper chain 
between $G_{i}$ and $G_{j}$ are all of dimension $d$, and hence belong to $\left\{G_{1}, \ldots, G_{s}\right\}$. Therefore $\Sigma=\left\langle G_{1}, \ldots, G_{s}\right\rangle$ is pure tree and connected in codimension 1. By Proposition 1.16. $\left\langle G_{1}, \ldots, G_{s}\right\rangle$ has at least two leaves.

We claim that at least one of the leaves of $\Sigma$ is a leaf of $\Delta$. Suppose this is not the case. We take any two leaves of $\Sigma$, say $G_{i}$ and $G_{j}$ with free vertex $x_{i}$ and $x_{j}$, respectively. Since $G_{i}$ and $G_{j}$ are not leaves in $\Delta$ there exist elements $F, F^{\prime} \in \mathcal{F}(\Delta) \backslash \mathcal{F}(\Sigma)$ with $x_{i} \in F$ and $x_{j} \in F^{\prime}$. Let $\mathcal{C}$ be a chain between $F$ and $F^{\prime}$. Since $\operatorname{dim} F<d$ and $\operatorname{dim} F^{\prime}<d$, all elements of this chain do not belong to $\mathcal{F}(\Sigma)$. On the other hand, let $\mathcal{C}^{\prime}$ be a proper chain between $G_{i}$ and $G_{j}$, then all elements of the chain belong to $\mathcal{F}(\Sigma)$, because $\operatorname{dim} G_{i}=\operatorname{dim} G_{j}=d$. Then the simplicial complex generated by the elements of these two chains has no leaf, a contradiction.

We may assume that $G_{i}$ is a leaf of $\Delta$. Removing $G_{i}$ from $\Delta$ yields a tree which is again connected in codimension 1 , and we may proceed as in case 1 .

Corollary 4.7. Let $\Delta$ be a 1-dimensional tree with facet ideal I. Then I has the alternating sum property.

Proof. It is clear that $\Delta$ is connected in codimension 1. The result follows from Theorem 4.6.

\section{REFERENCES}

[1] Bruns, W; Herzog, J. Cohen-Macaulay Rings, Revised version, Cambridge University Press, 1998.

[2] Eliahou, S; Villarreal, R.H. The second Betti number of an edge ideal, Aportaciones Matemáticas, Serie Comunicationes 1999, 25, 101-106.

[3] Faridi, S. The facet ideal af a simplicial complex. To appear in Manuscripta Mathematica.

[4] Faridi, S. Cohen-Macaulay properties of square-free monomial ideals. Preprint 2002.

[5] Fröberg, R. On Stanley-Reisner rings, Topics in algebra, Part 2 1990, 57-70.

[6] Stanley, R.P. Combinatorics and Commutative Algebra, Second Edition, Birkhäuser, 1996.

[7] Villarreal, R.H. Monomial Algebras, Marcel Dekker, Inc. 2001.

[8] Villarreal, R.H. Rees algebra of edge ideals, Comm. Algebra 1995, 23, 3513-3524.

[9] Villarreal, R.H. On the equations of the edge cone of a graph and some applications, Manuscripta Math, 1998, 97, 309-317. 